\title{
二芳基脯氨醇衍生物催化的硝基烯参与的多组分 不对称串联合成研究进展
}

\author{
严丽君 $a$ 徐菡 ${ }^{a}$ 王艳 $a$ 董建伟*,b 王永超*, $a$ \\ ( ${ }^{a}$ 云南师范大学职业技术教育学院 昆明 650092) \\ ( ${ }^{b}$ 曲靖师范学院化学与环境科学学院 云南曲靖 655011)
}

\begin{abstract}
摘要 硝基烯是一类重要的有机合成子，以硝基烯烃为原料，二芳基脯氨醇衍生物催化的多组分不对称串联反应是构 建复杂手性化合物的重要方法, 被广泛应用于有机合成和新药开发领域. 根据构建的目标化合物类型，较全面地总结 了基于二芳基脯氨醇衍生物催化、硝基烯为合成子的多组分不对称串联反应的合成研究，从反应的催化剂体系、反应 机理、实验结果、反应优点、存在的问题和局限性等方面进行介绍，并对今后的发展做出展望.
\end{abstract}

关键词 硝基烯; 二芳基脯氨醇衍生物; 多组分反应; 不对称合成; 串联反应

\section{Advances in Multicomponent Asymmetric Cascade Synthesis Involving Nitroolefin Catalyzed by Diarylprolinol Derivatives}

\author{
Yan, Lijun ${ }^{a} \quad \mathrm{Xu}, \operatorname{Han}^{a} \quad$ Wang, Yan ${ }^{a} \quad$ Dong, Jianwei ${ }^{*, b}$ Wang, Yongchao ${ }^{*, a}$ \\ ( ${ }^{a}$ College of Vocational and Technical Education, Yunnan Normal University, Kunming 650092) \\ $\left({ }^{b}\right.$ College of Chemistry and Environmental Science, Qujing Normal University, Qujing, Yunnan 655011)
}

\begin{abstract}
Nitroolefin is an important class of organic synthons. The synthetic method of multicomponent asymmetric cascade reactions involving nitroolefin catalyzed by diarlyprolinol derivatives is quite important for the construction of complex chiral compounds. It is widely used in organic synthesis and new drugs development. In this paper, the multicomponent asymmetric cascade synthesis involving nitroalkenes catalyzed by diarlyprolinol derivatives is comprehensively summarized based on the type of target compounds. In detail, the catalyst systems, reaction mechanisms, experimental results, reaction advantages, existing problems and limitations for this synthetic method are introduced respectively. The future development for this synthetic period is further evaluated as well.

Keywords nitroolefin; diarlyprolinol derivatives; multicomponent reactions; asymmetric synthesis; cascade reactions
\end{abstract}

有机催化多组分不对称串联反应是利用有机手性 小分子为催化剂, 通过烯胺活化、亚胺活化、氢键活化 等多种催化模式巧妙结合, 将三种或三种以上的原料经 “一锅法”反应实现多个化学键和多个手性中心的构建, 生成包含所有底物主要结构片段的一类手性新化合物 的反应过程 ${ }^{[1]}$. 这种高效的合成方法是有机不对称催化 反应和多组分串联反应的有机结合, 具有操作简洁、原 子经济性高、立体选择性好、产物结构多样、无需进行
保护和去保护操作及不需要中间体的分离纯化等优势, 是合成分子多样、结构复杂的手性化合物的新方法 ${ }^{[2]}$. 手性仲胺是催化多组分不对称串联反应的最有效催化 剂之一, 主要通过烯胺和亚胺活化, 结合氢键作用、位 阻效应等模式对反应物进行不对称催化 ${ }^{[3]}$. Hajos 等 ${ }^{[4]}$ 于 1971 年首次报道了 $L$-脯氨酸催化的不对称 Hajos/Parrish/Eder/Sauer/Wiechert 串联反应, 实现了手性仲胺在 有机催化多组分不对称串联反应中的首例应用。自

\footnotetext{
* Corresponding authors. E-mail: yongchaowang126@126.com; jwdongyn@mail.qjnu.edu.cn Received September 17, 2019; revised October 28, 2019; published online November 13, 2019.

Project supported by the Applied Basic Research Project of Yunnan Provincial Department of Science and Technology (Nos. 2017FD073, 2018FD016, 2018FD081), the Scientific Research Foundation of Yunnan Provincial Education Department (No. 2017ZZX075) and the Yunnan Local Colleges Applied Basic Research Project (No. 2017FH001-092).

云南省科技厅应用基础研究基金(Nos. 2017FD073, 2018FD016, 2018FD081)、云南省教育厅科学研究基金(No. 2017ZZX075)和云南地方高校应用基础 研究基金(No. 2017FH001-092)资助项目.
} 
2000 年以来, 手性胺在不对称催化串联反应领域中取 得了重大进展, 大量的新型手性胺催化剂及催化反应体 系被不断地发展和完善. 2005 年, Marigo ${ }^{[5]}$ 和 Hayashid 等 ${ }^{6]}$ 分别报道了二苯基脯氨醇硅醚的合成及其在不对称 串联反应中的催化应用, 该类反应取得了优异的催化活 性、突出的立体选择性和良好的底物普适性, 极大地促 进了有机催化不对称合成研究的发展 ${ }^{[7]}$. 硝基烯烃是一 类重要的有机合成子, 其硝基的拉电子效应使双键易与 亲核试剂(如碳负离子、亚磷酸醋等)发生加成反应, 在 有机合成中被广泛研究应用 ${ }^{[8]}$. 此外, 加成产物的硝基 还可以进一步转化成胺、弜、羟胺、腈、醇、醛、酮和 杂环等多种具有显著生物活性的重要化合物 ${ }^{[9]}$.

近年来, 以硝基烯为合成子、二芳基脯氨醇衍生物 (1)为催化剂的多组分不对称串联反应受到了普遍关注, 基于多组分 “一锅法” 不对称串联反应能实现多个新的 化学键的构建和多个手性中心的手性控制, 能为有机合 成方法学研究、复杂天然产物的合成及新药开发提供新 思路. 本文从合成手性吲哚衍生物、多取代手性环已烯 醛衍生物、手性哌啶衍生物、其他类型手性化合物几个 方面, 对以硝基烯为合成子、二芳基脯氨醇衍生物为催 化剂的多组分不对称串联反应的催化体系、反应的主要 特点、催化机理等方面进行阐述, 并对其应用研究进行 了展望.

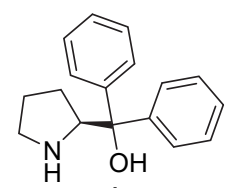

$1 \mathrm{a}$

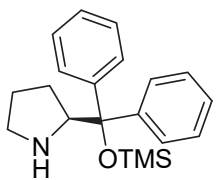

$1 \mathrm{~b}$

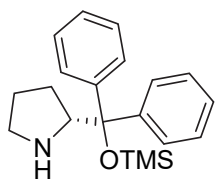

$1 \mathrm{c}$
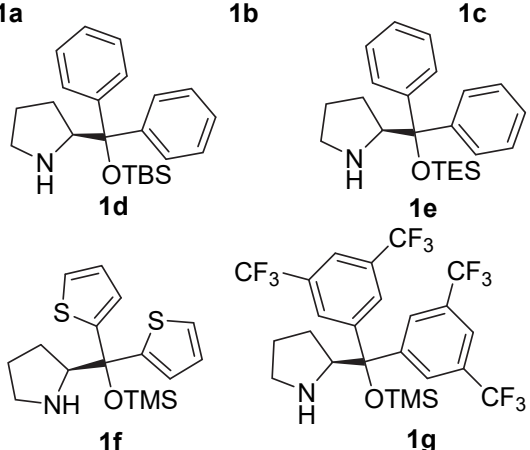

\section{1 手性吲哚衍生物的不对称合成}

\section{1 手性 3,3'-螺环氧化吲哚衍生物的不对称合成}

手性 3,3'-螺环氧化吲哚(2)是一类重要的含氮杂环 化合物, 其分子结构中包含稳定的手性多环体系, 具有 良好的构象约束性, 能与多种生物蛋白进行精确识别与 严格的手性匹配, 广泛存在于天然生物碱及生物活性分 子中 ${ }^{[10]}$, 具有抗肿瘤、抗 HIV、抗氧化、抗肺结核、抗 菌等多种生物活性 ${ }^{[11]}$. 近年来, 通过有机催化不对称反
应来构建手性 3,3'-螺环氧化吲哚衍生物的研究受到化 学及药学工作者的普遍关注, 并取得了重大的突破 ${ }^{[2]}$. 然而，建立高效、简洁和立体选择性优异的新反应，并 应用于新型手性 3,3'-螺环氧化吲哚衍生物的合成仍是 有机及药物合成研究领域的热点课题.
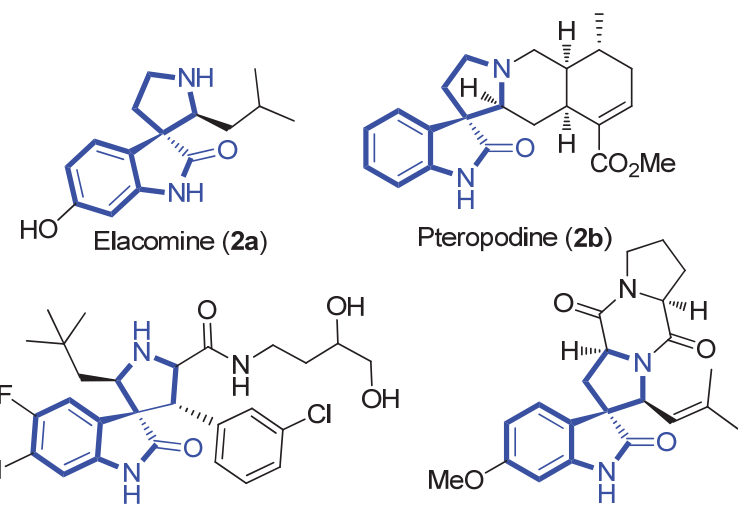

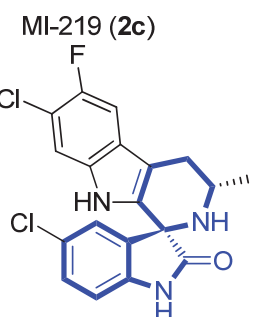

NITD609 (2e)
Spirotryptostatin A (2d)

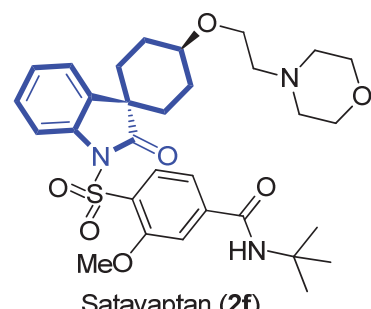

以硝基烯为反应合成子，通过多组分不对称串联反 应来构建手性螺环吲哚衍生物的合成方法取得了一系 列进展. 2010 年, 陈应春研究组 ${ }^{[13]}$ 报道了二苯基脯氨醇 甲基硅醚(1b)催化的三组分不对称 $[2+2+2]$ 环化反应, 以 $N$-Boc 氧化吲哚衍生物 3a、脂肪醛(4a)为起始原料, 在催化剂 $1 \mathbf{b}$ 作用下得到不对称 Michael 加成中间体 $\mathbf{I}$, 再进一步与硝基烯化合物 5a 发生串联环化反应，以 $82 \% \sim 90 \%$ 的产率和 $90 \% \sim 95 \% e e$ 的对映异构体选择性 构建了一系列具有六个手性中心的螺环氧化吲哚衍生 物 6 (Eq. 1). 该研究为手性螺环吲哚类先导化合物的发 现奠定了基础，然而该方法不适用于脂肪硝基烯参
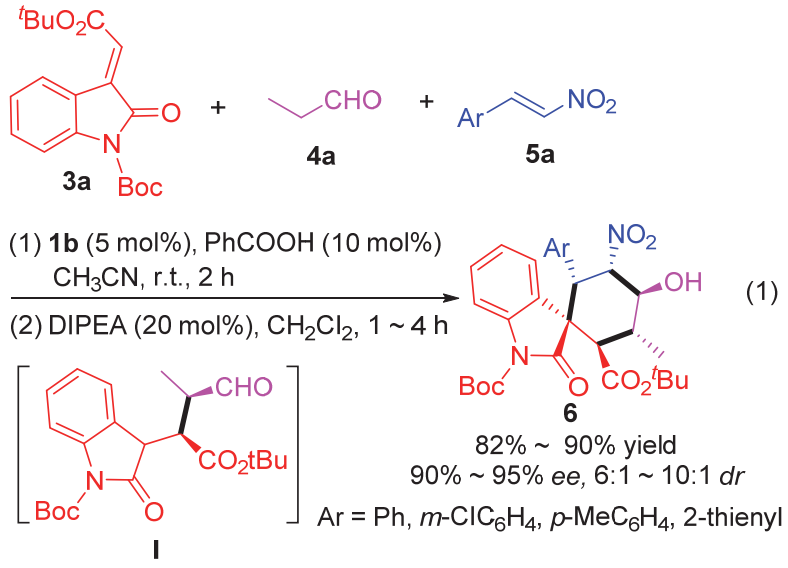
与的不对称串联反应，这在一定程度上限制了该方法的 普适性.

同年, 该研究组又探究了 3-烯基氧化吲哚(7)、肉桂 醛 (8a) 和硝基苯乙烯 $(5 b)$ 的三组分不对称 Michael/Michael/Michael/aldol 串联反应，该方法基于二苯基脯氨 醇甲基硅醚(1b)对反应底物的亚胺离子-烯胺-亚胺离 子-烯胺的四重活化, 以优异的非立体选择性( $>99 \%$ $d e)$ 和立体选择性( $>99 \% e e)$ 合成了一系列 3-螺环氧化 吲哚衍生物 $\mathbf{9}^{[14]}$ (Eq. 2). 该方法通过简洁的多组分不对 称串联反应，实现了八个手性中心的控制和手性螺环、 稠环的构建.

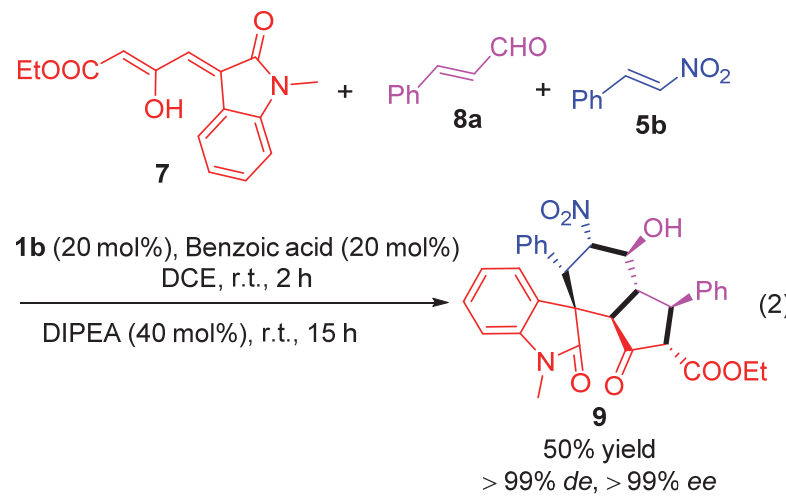

彭成研究组 ${ }^{[15]}$ 于 2012 年开展了硝基烯参与的三组 分不对称 Michael/aldol/缩醛化串联反应构建手性 3,3'螺吡喃氧化吲哚衍生物的方法学研究(Eq. 3), 取得了优 异的立体选择性( $94 \%$ ～94\% ee). 该方法通过二苯基脯 氨醇甲基硅醚(1b)对脂肪醛 (4)进行活化发生不对称 Michael 加成, 得到的加成产物 $\mathbf{I}$ 不需分离, 直接与吲哚 醌(10)发生分子间 Henry 反应得到中间体 II, II 再通过手 性胺(1b)催化, 发生分子内缩醛化反应得到目标化合物 11. 在该反应中, 手性催化剂 1b 两次对醛基进行活化,
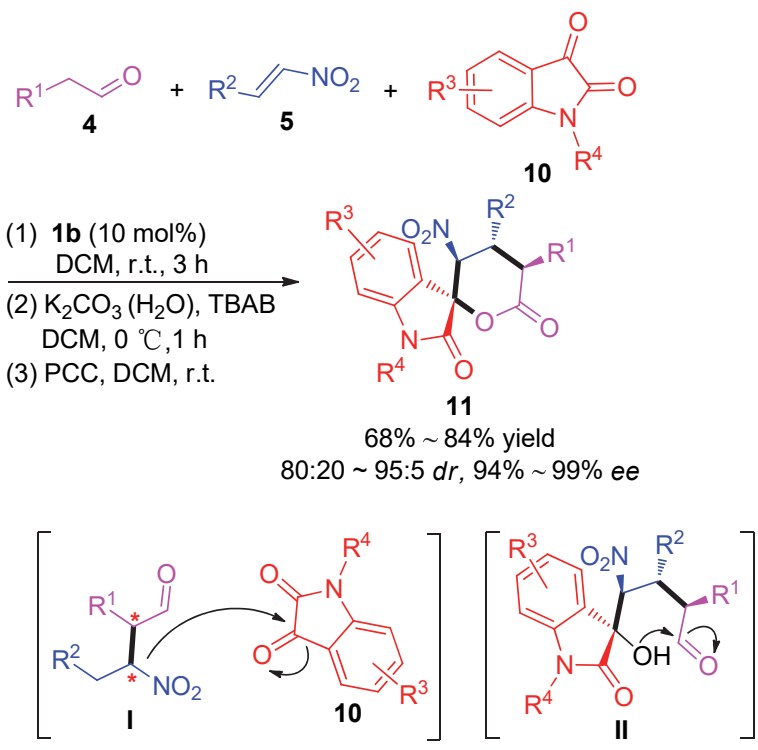

实现对目标化合物立体选择性的控制，具有单分子多次 催化活化的作用.

2013 年, 周兵等 ${ }^{[16]}$ 报道了手性仲胺-硫艮双催化剂 协同催化的多组分不对称 Michael/ Michael/aldol 串联加 成反应，经 “一锅法” 串联反应实现了三个新的 $\mathrm{C}-\mathrm{C}$ 键、五个连续手性中心(含一个手性季碳中心)的构建. 该方法利用二苯基脯氨硅醚 $(\mathbf{1 b}$ 或 1d)和双功能硫脲-奎 宁(13a)为催化剂, 以 $N$-Boc-2-吲哚酮(12)、硝基烯化合 物(5)和 $\alpha, \beta$-不饱和醛 $(\mathbf{8})$ 为原料, 以良好的产率和优异 的对映异构体选择性合成了两类结构相似的手性螺环 吲哚衍生物 14, 15 (Scheme 1). 研究发现当把二苯基脯 氨甲基硅醚(1b)换成二苯基脯氨叔丁基二甲基硅醚(1d) 后, 可以得到 3-螺环手性构型翻转的吲哚衍生物. 从反 应机理分析看出, 双功能硫脲-奎宁(13a)通过氢键作用 对硝基烯化合物进行活化，同时 13a 的叔胺结构作为 Brønsted 碱可以诱导 $N$-Boc-2-吲哚酮(12)酰胺结构的烯 醇化，促进其与硝基烯化合物 $\mathbf{5}$ 的不对称 Michael 加成 (TS-1). 二苯基脯氨硅醚则对 $\alpha, \beta$-不饱和醛 8 通过亚胺 催化与 Michael 加成产物发生分子间 nitro-Michael 加成, 并经碱催化发生分子内 aldol 反应(TS-2)最终构建目标 化合物 14 和 15. 该方法实现了催化剂控制的产物手性 翻转, 具有重要的研究意义.

谭睿课题组 ${ }^{[17]}$ 于 2016 年开发了三组分 $[2+2+2]$ 串 联环化反应，通过二苯基脯氨醇甲基硅醚(1b)催化的不 对称 Michael/Michael/Henry 串联反应，合成了一系列具 有七个连续手性中心的 3,3'-螺环氧化吲哚类衍生物 17 (Eq. 4). 反应从 $N$-Boc 氧化吲哚烯(3)、邻羟基内醚(16) 出发, 基于烯胺活化发生分子间不对称 Michael 加成, 其加成产物再与 $\beta$-硝基烯烃 5 发生 Michael/Henry 串联 反应，以 39\% 99\%的产率和 92\% 99\% ee 的对映异构 体选择性实现多个 $\mathrm{C}-\mathrm{C}$ 的构建和多个手性中心的高效 控制。该反应具有高效、选择性好、环境友好等特点，有 潜在的开发应用价值.

Chaudhari 等 ${ }^{[18]}$ 于 2017 年开发了有机不对称催化的 三组分 Michael/Michael/Michael/aldol 串联反应，合成了 两类多取代 3,3'螺环氧化吲哚衍生物 19 和 20 . 该反应 以二苯基脯氨醇硅醚(1b 或 $1 \mathrm{~d})$ 为催化剂, 经硝基烯吲哚 酮 18 和两分子的 $\alpha, \beta$-不饱和醛 $8 \mathrm{~b}$ 发生三组分不对称四 级串联反应，以 $55 \% \sim 64 \%$ 的产率和优异的对映异构体 选择性实现对多个手性碳的立体选择性控制 (Scheme 2). 该方法通过改变催化剂的芳环结构，可以实现螺环 吲哚 3-位碳的手性翻转, 反应经历了两种可能的过渡态 (TSA 和 TSB $\sim$ TSC), 导致化合物 19 和 20 具有不同的 手性构型. 
<smiles>[R]C(=O)N1C(=O)[C@@]2(c3cc([R])ccc31)[C@@H](O)C[C@@H]([R])[C@H]([N+](=O)[O-])[C@@H]2O</smiles>

15 samples $45 \% \sim 92 \%$ yield, $90 \% \sim 99 \%$ ee<smiles>C=CC1CN2CC1CC2[C@H](NC(=S)Nc1cc(C(F)(F)F)cc(C(F)(F)F)c1)c1ccnc2ccc(OC)cc12</smiles>

$13 a$<smiles>[R]C=C[N+](=O)[O-]</smiles>
$13 a+1 d$ Toluene, r.t. Overnight<smiles>[R]c1ccc2c(c1)[C@]1(C(=O)N2C(C)(C)C)C([R])C([N+](=O)[O-])CC[C@H]1[R]</smiles>
20 samples $32 \% \sim 94 \%$ yield, $>99 \%$ ee

图式 1 有机不对称催化三组分 Michael/Michael/aldol 串联反应合成手性螺环吲哚衍生物 14 和 15

Scheme 1 Synthesis of chiral spirooxindole derivatives 14 and 15 by asymmetric organocatalytic Michael/Michael/aldol cascade reaction

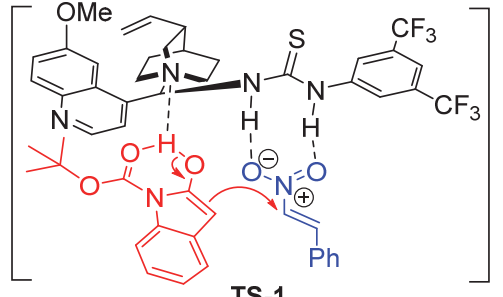

TS-1

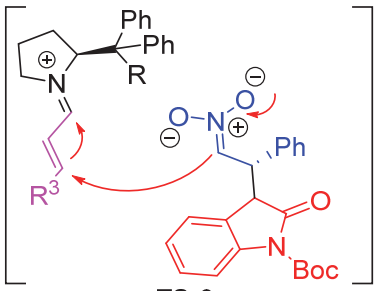

TS-2<smiles>[R]C=C1C(=O)N(C(C)(C)C)c2cc[R1]([H])cc21</smiles>

3

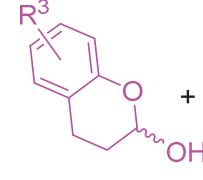

16
(1) 1 b (5 mol\%), 4- $\mathrm{NO}_{2} \mathrm{C}_{6} \mathrm{H}_{4} \mathrm{COOH}$ (5 mol\%)<smiles>[R]C=C[N+](=O)[O-]</smiles>

(2) Base

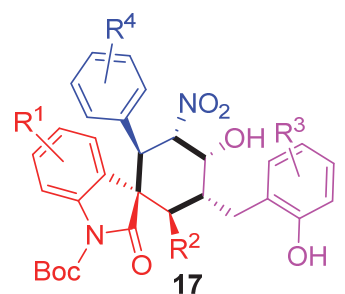

23 samples $39 \% \sim 99 \%$ yield, $92 \% \sim 99 \%$ ee

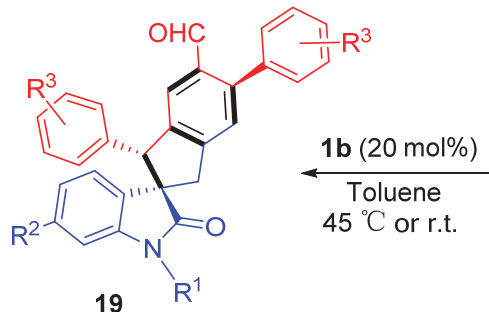

up to $97 \%$ ee<smiles>[R]c1ccc2c(c1)N([R1])C(=O)C2C/C=C\[N+](=O)[O-]</smiles>

$\mathrm{R}^{1}=\mathrm{Me}, \mathrm{H}$ $\mathrm{R}^{2}=\mathrm{H}, \mathrm{Cl}$

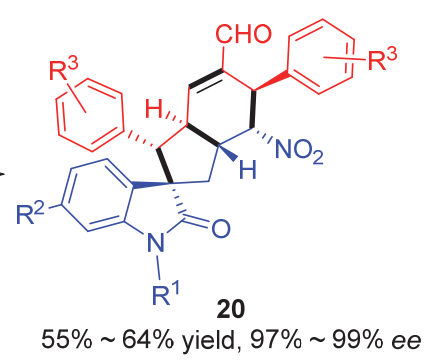

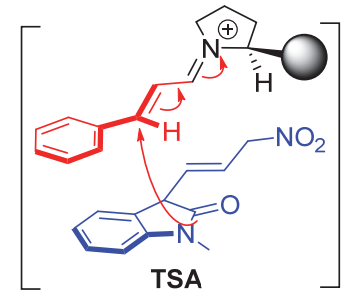
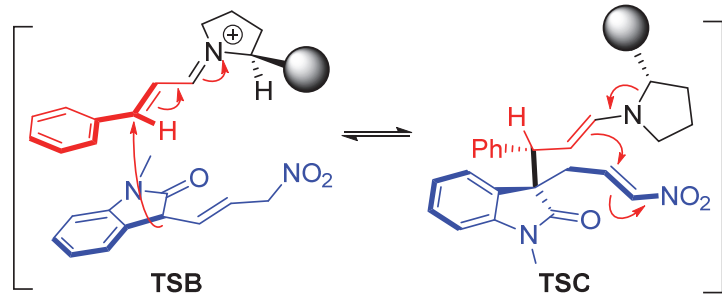

图式 2 三组分不对称 Michael/Michael/Michael/aldol 串联反应

Scheme 2 Asymmetric organocatalytic three-component Michael/Michael/Michael/aldol cascade reaction

\section{2 其它手性吲哚衍生物的不对称合成}

吲哚喹嗪衍生物是一类重要的吲哚类生物碱, 具有 多种药用活性, 基于有机催化的不对称串联反应构建手 性吲哚喹嗪骨架具有重要的研究价值 ${ }^{[19]}$. 2014 年, 林国 强研究组 ${ }^{[20]}$ 建立了二苯基脯氨醇甲基硅醚(1b)催化的两 分子脂肪醛 4、硝基烯(5)和色胺(21)的四组分不对称
Michael/aza-Henry/Pictet-Spengler 串联反应，以优异的 立体选择性(>20：1 $d r, 91 \% \sim 98 \% e e)$ 合成了 17 个多 取代吲哚喹嗪衍生物 22 (Scheme 3). 在该反应中, 一分 子脂肪醛 4 和硝基烯(5)首先通过手性仲胺催化发生不 对称 Michael 加成得到中间体 I-1, 随后与亚胺中间体 $\mathbf{I}-2$ 发生不对称 $a z a$-Henry 反应获得中间体 I-3, 随后依 
次发生缩氨化反应、脱水消除得到亚胺离子中间体 I-4, 最后发生分子内 Pictet-Spengler 反应合成化合物 22. 该 研究首次实现了色胺参与的不对称 $a z a$-Henry 反应, 并 通过四组分 “一锅法” 不对称串联反应构建了五个连续 的手性中心.

2010 年, Enders 等 ${ }^{[21]}$ 将吲哚(23)、硝基烯(5)和两分 子丙烯醛 $(\mathbf{8 c})$ 的四组分 “一锅法” 不对称催化串联反应 应用于合成多手性中心的吲哚衍生物, 以 $23 \% \sim 82 \%$ 的 产率和优异的立体选择性(91：9～95：5 $d r, 94 \% \sim 99 \%$ $e e$ )合成了一系列 3-环己烯基甲基吲哚 24 (Scheme 4). 该反应基于二苯基脯氨醇甲基硅醚 (1b) 催化的 Friedel-Crafts-type/Michael/Michael/aldol 串联反应, 实 现了四个新的 $\mathrm{C}-\mathrm{C}$ 键的构建和多个手性中心的控制. 在该不对称串联反应中, 手性仲胺首先形成亚胺离子对 一分子丙烯醛 (8c) 进行活化, 进而与吲哚 (23) 发生 Friedel-Crafts-type 反应得到烯胺中间体 I, I 再与硝基烯
烃 5 发生分子间不对称 Michael 加成得到反应中间体 II, II 再经水解作用、与亚胺离子的分子间不对称 Michael 加成, 得到的加成产物 III 再通过分子内不对称 aldol 反 应得到化合物 24. 该方法实现了多个新的 $\mathrm{C}-\mathrm{C}$ 键的构 建，并形成了多取代手性环已烯醛结构单元.

\section{2 手性环己烯醛衍生物的不对称合成}

\section{1 单环手性环己烯醛衍生物的不对称合成}

单环手性环己烯醛是合成天然产物及手性药物分 子的重要原料或关键中间体，手性中心的立体选择性控 制是合成该类化合物的关键环节 ${ }^{[22]}$. Enders 研究组 ${ }^{[23]}$ 于 2008 年报道了手性仲胺催化的三组分不对称 Michael/ Michael/aldol 串联反应，以 $18 \% \sim 60 \%$ 的产率、良好的 立体选择性(65：35～89：11 dr, 97\% 99\%ee)合成了 一系列多取代环已烯醛衍生物 25 (Scheme 5). 反应以二

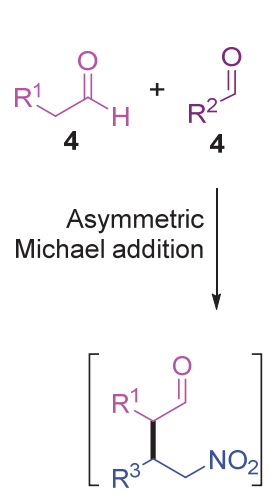

I-1

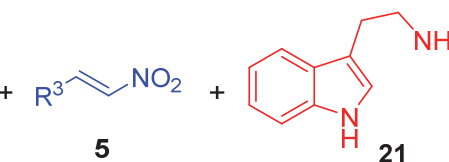

5

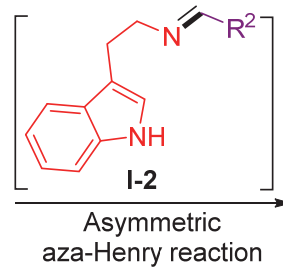
aza-Henry reaction

(1) $\mathbf{1 b}(5 \mathrm{~mol} \%), p$-nitrophenol $\mathrm{CH}_{2} \mathrm{Cl}_{2}, 0{ }^{\circ} \mathrm{C}$ or r.t.

\section{(2) $\mathrm{DABCO},-40^{\circ} \mathrm{C}, 22 \mathrm{~h}$}

(3) TMG, r.t., $12 \mathrm{~h}$

(4) $\mathrm{TsOH} \cdot \mathrm{H}_{2} \mathrm{O}$, r.t., $4 \mathrm{~h}$

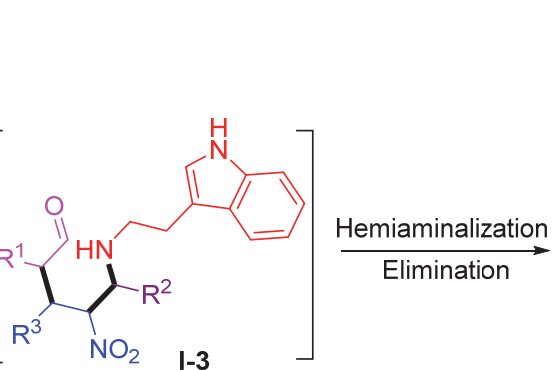

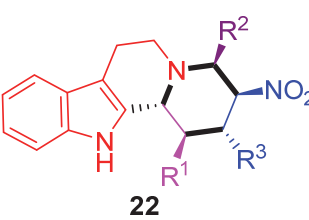

17 samples

$30 \% \sim 55 \%$ yield $>20: 1 d r, 91 \% \sim 98 \%$ ee

Pictet-Spengler

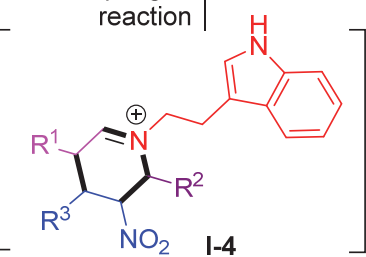

图式 3 四组分不对称串联反应合成手性吲槑喹嗪衍生物 22

Scheme 3 Synthesis of chiral indoloquinolizidine derivatives (22) via four-component asymmetric cascade reaction<smiles>[R]1ccc2[nH]ccc2c1</smiles>

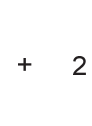<smiles>O=CC=O</smiles><smiles>[R]C=C[N+](=O)[O-]</smiles>

5<smiles>[R1]c1ccc2[nH]cc(C/C=C\N3CCCC3C(O[S+](C)(C)C)(c3ccccc3)c3ccccc3)c2c1</smiles>

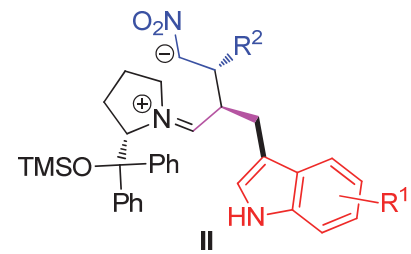

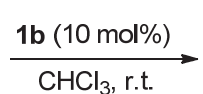

9 samples, $23 \% \sim 82 \%$ yield

91:9 95:5 dr, 94\% 99\% ee $\quad \mathrm{NO}_{2}$

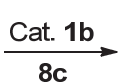

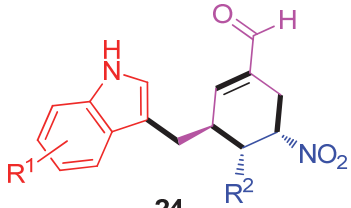

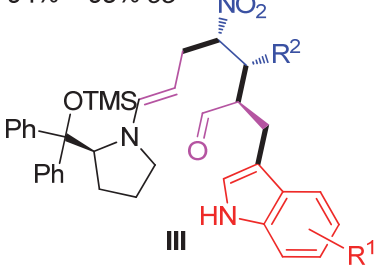

图式 4 有机催化不对称四组分 Friedel-Crafts-type/Michael/Michael/aldol 串联反应

Scheme 4 Asymmetric organocatalytic four-component Friedel-Crafts-type/Michael/Michael/aldol cascade reaction 


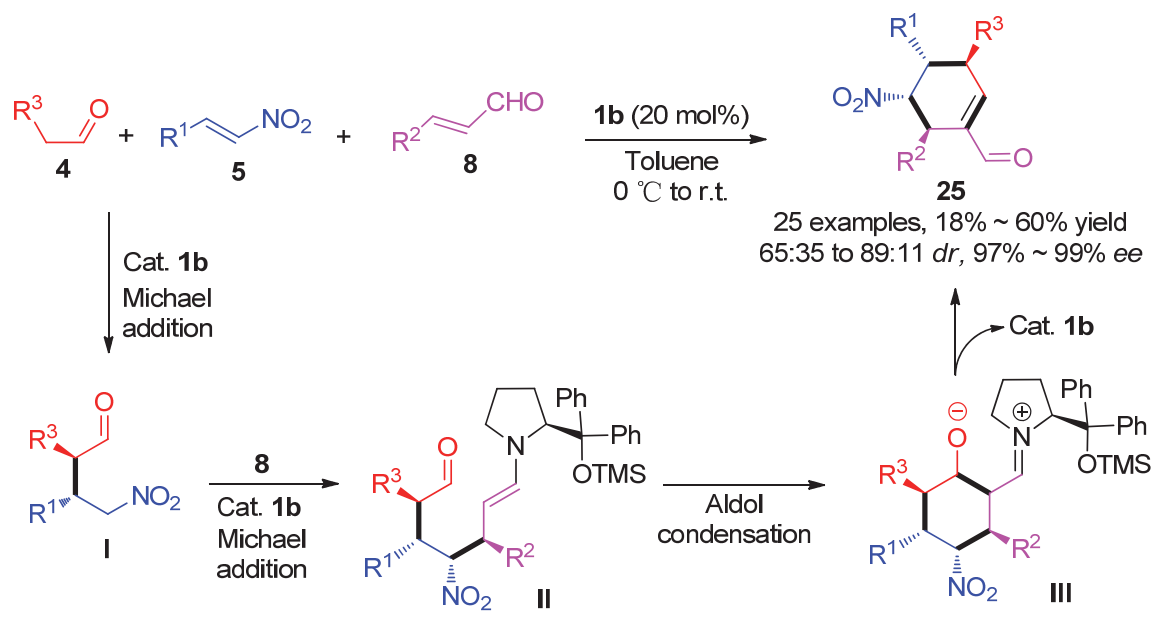

图式 5 有机催化不对称 Michael/Michael/aldol 串联反应合成环已烯醛衍生物 $\mathbf{2 5}$

Scheme 5 Synthesis of chiral cyclohexene-carbaldehyde derivatives 25 by asymmetric organocatalytic Michael/Michael/aldol cascade reaction

苯基脯氨醇甲基硅醚(1b)为催化剂, 先发生脂肪醛 4 和 硝基烯(5)的不对称 Michael 加成, 加成产物 $\mathbf{I}$ 再与 $\alpha, \beta$ 不饱和醛 8 进行不对称 Michael 加成, 得到的中间体 II 再经分子内 aldol 缩合得到中间体 III, 从而实现化合物 25 的合成和催化剂的再生. 该研究组还进一步探索了 基于分子内不对称 Diels-Alder 反应构建具有八个手性 中心的三环化合物的合成研究, 实现了多个 $\mathrm{C}-\mathrm{C}$ 的构 建和多个手性中心的高效控制 ${ }^{[24]}$.

2009 年, 龚跃法研究组 ${ }^{[25]}$ 通过四组分不对称 $o x a-$ Michael/Michael/Michael/aldol 串联反应成功合成了一系 列手性环己烯醛衍生物 26. 反应基于二苯基脯氨醇甲 基硅瞵(1b)的亚胺离子催化,一分子丙烯醛 $(8 \mathrm{c})$ 与醇 27 发生不对称 oxa-Michael 加成, 加成产物再经烯胺活化, 与硝基烯(5)进行不对称 Michael 加成得到中间体 I, I 再 通过 $1 \mathrm{~b}$ 的亚胺离子催化, 与第二分子的丙烯醛 $(8 \mathrm{c})$ 发生 不对称 Michael 加成, 得到的中间体 II 再经分子内不对 称 aldol 反应, 以优异立体选择性( $>20: 1 d r,>99 \% e e)$ 合成了 13 个手性环已烯醛衍生物 26 (Scheme 6). 该方 法基于三个连续的 Michael 加成及一个分子内 aldol 反应 串联反应, 构建了四个新的 $\mathrm{C}-\mathrm{C} / \mathrm{O}$ 键, 发展了一种高 效、简洁、原子经济性好的不对称合成方法.

2010 年, Enders 研究组 ${ }^{[26]}$ 开发了微波辅助的四组分 不对称 Michael/Henry/Michael/aldol 串联反应. 反应以 二苯基脯氨醇甲基硅醚(1b)为催化剂, 通过硝基烯烃(5) 和三分子乙醛 $(\mathbf{4 b})$ 的串联反应合成了三取代环已烯酫 28 (Eq. 5). 研究表明一定量的水能促进反应的进行, 而 一定范围内的升温并不会影响反应的立体选择性, 作者 基于 ESI-MS 实验探究了反应机理及催化循环过程. 该 方法具有反应条件温和、绿色、简洁等优点.

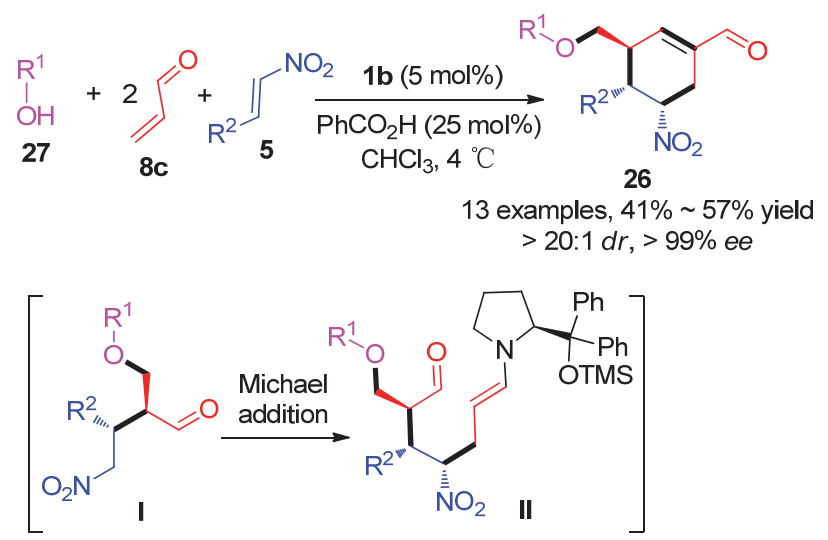

图式 6 有机催化不对称四组分 oxa-Michael/Michael/Michael/ aldol 串联反应

Scheme 6 Asymmetric organocatalytic four-component oxaMichael/Michael/Michael/aldol cascade reaction

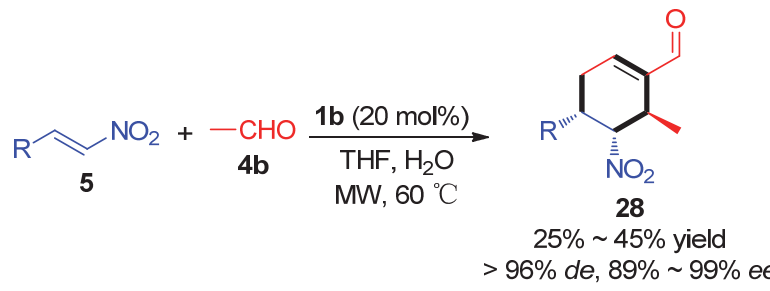

随后，Rueping 等 ${ }^{[27]}$ 受生物酶催化启发，建立了有 机催化的三组分不对称串联反应. 与酶促反应类似，该 方法可以通过控制反应物 $(\alpha, \beta$-不饱和醛)的浓度, 高选 择性地获得不同级的串联反应产物. 基于二苯基脯氨醇 甲基硅醚(1b)和 Hantzsch 二氢吡啶(HEH)协调催化的亚 胺离子一烯胺一亚胺离子一烯胺节联活化, 发生两分子的 $\alpha, \beta$-不饱和醛 (8) 和硝基烯(5)的不对称氢化/Michael/ Michael/aldol 串联反应，以中等的产率(35\% 61\%)和优 异的对映异构体选择性( $98 \%$ \% $99 \%$ ee) 合成了一系列环 
己烯醛衍生物 29 (Eq. 6).

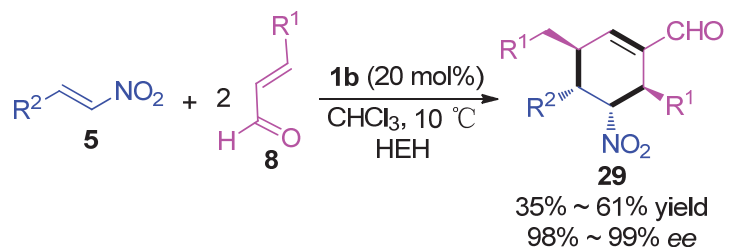

同年, 王锐研究组 ${ }^{[28]}$ 利用二噻吩 $L$-脯氨醇甲基硅 醚(1f)为催化剂, 探究了脂肪醛 (4)、 $\alpha, \beta$-不饱和醛 $(8)$ 和硝 基烯(5)的三组分不对称三级串联反应(Eq. 7). 该反应以 水为溶剂、十二烷基苯磺酸钠(SDBS)为表面活性剂, 在 温和、绿色环保的条件下实现了多手性中心和多个新的 $\mathrm{C}-\mathrm{C}$ 键的构建, 获得高手性纯的环己烯醛衍生物 $\mathbf{3 0}$ (76：24 90：10 dr, >99\%ee). 该反应在进行 $48 \mathrm{~h}$ 之 后，只取得了中等程度的产率 $(16 \% \sim 56 \%)$, 其可能的 原因是反应物及催化剂在水中的溶解性较差所致. 因 此, 表面活性剂的优化选择、反应温度的优化或许是该 方法值得进一步探究的方面.

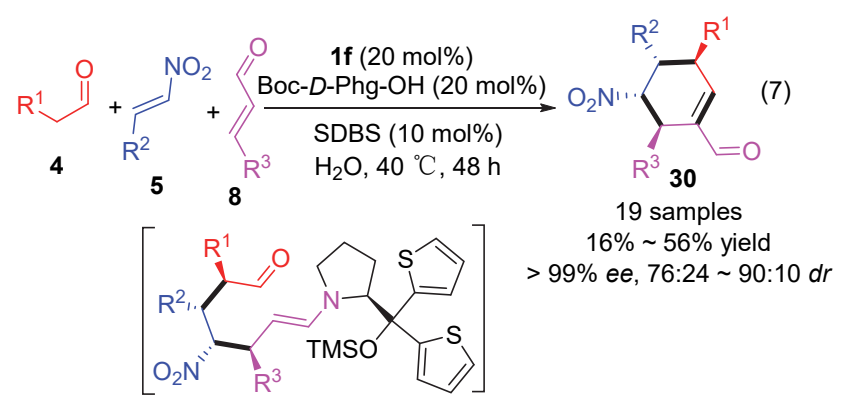

随后, Enders 研究组 ${ }^{[29]}$ 进一步探究了手性仲胺在多 组分不对称串联反应中的催化应用, 并于 2013 年报道 了一例四组分不对称 Friedel-Crafts/Michael/Michael/ aldol 新反应(Eq. 8). 通过二苯基脯氨醇甲基硅醚(1b)催 化的间甲氧基苯胺衍生物(31)、丙烯醛 $(\mathbf{8 c}) 、 \alpha, \beta$-不饱和 醛 $(8)$ 和硝基烯烃 $(5)$ 的四组分串联反应, 以良好的非对 映异构体选择性( $>95: 5 d r)$ 和优异的对映异构体选择 性(94\% 99\% ee) 获得了一类高度功能团化的环己烯醛 衍生物 32. 该方法使用了三种不同的 Michael 受体参与

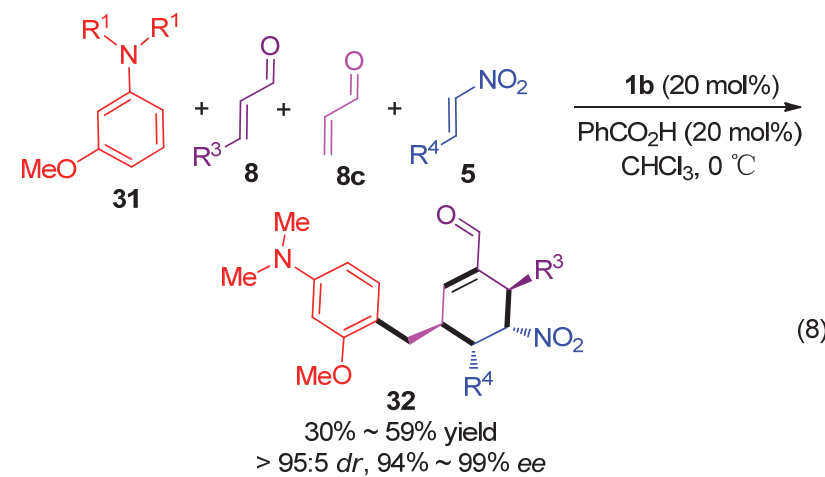

串联反应，表现出良好的普适性，其反应产物在药物研 究和农药开发领域具有潜在的应用价值.

Endes 等 ${ }^{[30]}$ 继续开发有机催化多组分串联反应，并 于 2015 年建立了基于二苯基脯氨醇甲基硅醚(1b)催化 的烯烃衍生物 33、两分子的丙烯醛(8c) 和硝基烯烃(5) 的四组分不对称 Vinylogous Friedel-Crafts/Michael/Michael/aldol 串联反应(Eq. 9). 该反应在温和的条件下，以 中等的产率(30\% 69\%)和突出的选择性(9: 1 20:1 $d r,>99 \% e e)$ 合成了一系列具有三个连续手性中心的 环己烯醛衍生物 34. 该方法实现了四个 $\mathrm{C}-\mathrm{C}$ 新键的构 建，具有良好的原子经济性.

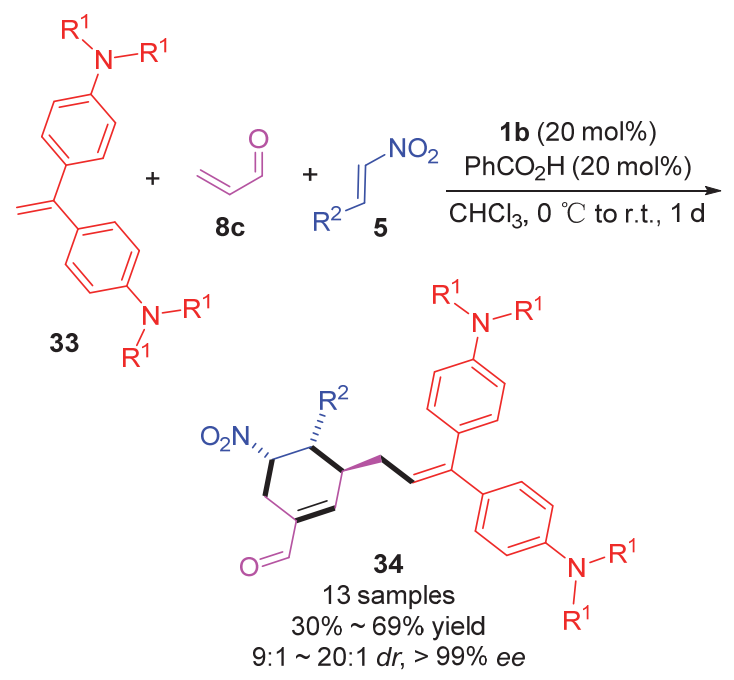

\section{2 手性三环色满烯醛衍生物的不对称合成}

手性三环色满烯醛衍生物是一类重要的多环化合

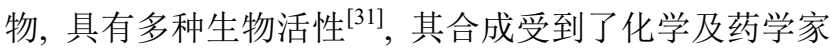
的普遍关注. 2009 年, 洪伯诚研究组 ${ }^{[32]}$ 首次报道了多组 分不对称 oxa-Michael/Michael/Michael/aldol 四级串联反 应在有机合成中的应用. 反应以二苯基脯氨醇甲基硅醚 (1b)为催化剂, 以反式-2-(2-硝基乙烯基)苯酚(35a)和 $\alpha, \beta$-不饱和醛 $(\mathbf{8 d}, 8 \mathrm{e})$ 为原料, 在 $25{ }^{\circ} \mathrm{C}$ 的条件下合成了 含有五个手性中心的四氢- $6 H$-苯并 $[c]$ 苯并吡喃化合物 36, 取得了优异的立体选择性(Scheme 7). 该反应优异 的立体选择性可能与不对称 $o x a$-Michael 加成有关，反 应首先在二苯基脯氨醇甲基硅醚(1b)的催化作用下，取 得高手性纯的 Michael加成产物 $\mathbf{I}$, 然后再发生分子内不 对称双 Michael 加成和不对称 aldol 串联反应，从而合成 了具有多个手性中心的三环体系结构的化合物 36. 该 研究对复杂手性多环化合物的合成具有重要的指导意 义.

随后，该研究组进一步探究了三组分不对称 $o x a-$ Michael/Michael/Michael/aldol 串联反应在天然产物全合 成中的应用，并实现了天然产物 $(+)-C o n i c o l$ 的首次不 


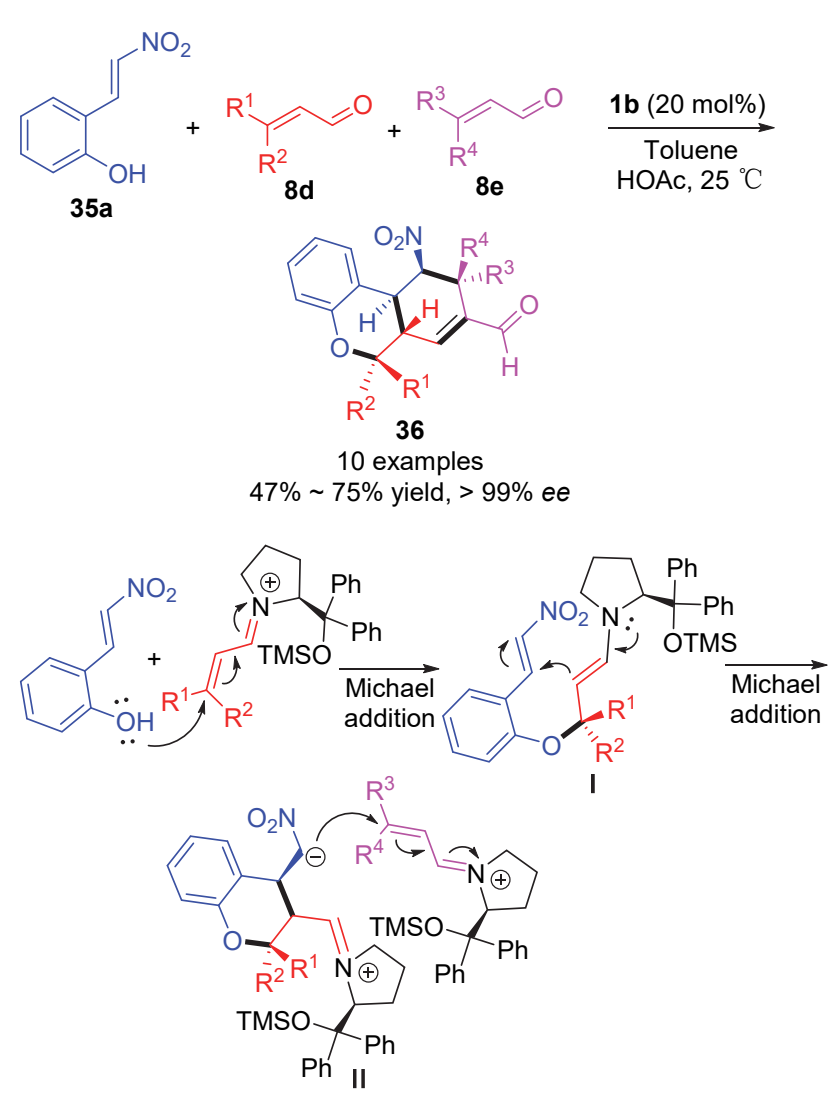

图式 7 有机催化不对称 oxa-Michael/Michael/Michael/aldol 串 联反应

Scheme 7 Asymmetric organocatalytic oxa-Michael/Michael/ Michael/aldol cascade reaction

对称全合成 ${ }^{[33]}$. 该反应基于二苯基脯氨醇甲基硅醚(1b) 催化的 2-(反式-2-硝基乙烯基)-苯-1,4-二醇(35b)、异戊 烯醛 $(\mathbf{8 f})$ 和 4,4-二甲氧基-2-丁烯醛 $(\mathbf{8 g})$ 的三组分不对称 oxa-Michael/Michael/Michael/aldol 串联反应, 以优异的 对映异构体选择性(>99\% ee) 合成了关键中间体 37, 再 经几个步骤实现 $(+)$-Conicol (38) 的不对称全合成 (Scheme 8), 为该类化合物的进一步生物活性研究奠定 了基础.

Kumar 等 ${ }^{[34]}$ 于 2017 年建立了二苯基脯氨醇甲基硅 醚(1b)催化的硝基苯并呋喃(39)、脂肪醛(4)和 $\alpha, \beta$-不饱 和醛(8)的三组分不对称 Michael/Michael/aldol 串联反应 (Eq. 10). 反应在温和的条件下，以中等的产率 $(20 \%$ 66\%)和优异的立体选择性合成了 25 个具有四个手性中 心的三环色满衍生物 $\mathbf{4 0}$. 当反应放大到克量级时仍能 保持理想的立体选择性, 以 $42 \%$ 的产率、优异的立体选 择性(9：1 20：1 $d r, 99 \% e e$ )合成了 $0.87 \mathrm{~g}$ 的三环色满 衍生物. 此外, 该反应得到的三环色满衍生物还可发生 醛基的 $\mathrm{NaBH}_{4}$ 还原、Wittig 反应等, 在有机合成和新药 开发领域具有潜在的应用价值.

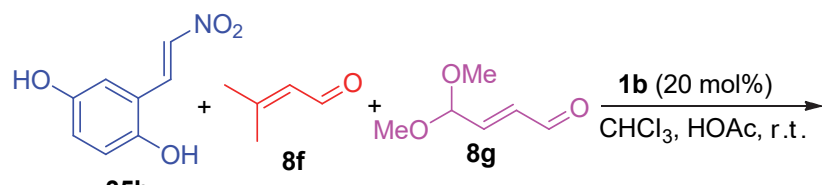

35b

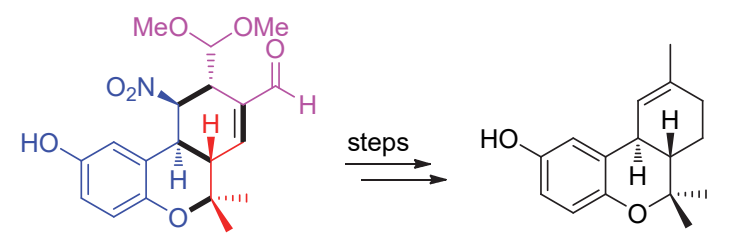

$69 \%$ yield, $>99 \%$ ee

(+)-Conicol (38)

图式 8 基于有机催化三组分 oxa-Michael/Michael/Michael/ aldol 串联反应的不对称全合成

Scheme 8 Asymmetric total synthesis via organocatalytic threecomponent oxa-Michael/Michael/Michael/aldol cascade reaction

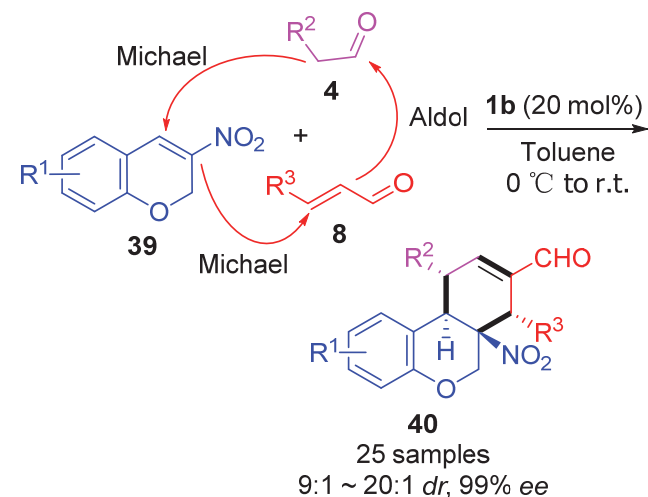

为进一步探索合成手性三环色满衍生物的新方法, Kumar 等 ${ }^{[35]}$ 于 2018 年报道了 3-硝基-2H-苯并吡喃(39)、 两分子的丙烯醛 $(\mathbf{8 c})$ 和醇 $(27)$ 的四组分不对称 $o x a-\mathrm{Mi}-$ chael/Michael/Michael/aldol 串联反应(Eq. 11). 反应以 10 $\mathrm{mol} \%$ 的二苯基脯氨醇甲基硅醚(1b)为催化剂、0 ${ }^{\circ} \mathrm{C}$ 条件 下，以 $30 \% \sim 70 \%$ 的产率、优异的立体选择性( $>20: 1$ $d r,>99 \% e e$ ) 合成了 22 个手性三环色满衍生物 41. 该 方法通过 “一锅法” 四组分不对称串联反应，高效地实 现了四个新的 $\mathrm{C}-\mathrm{C} / \mathrm{O}$ 键的构建、四个手性中心的手性 控制，并具有良好的底物多样适用性.

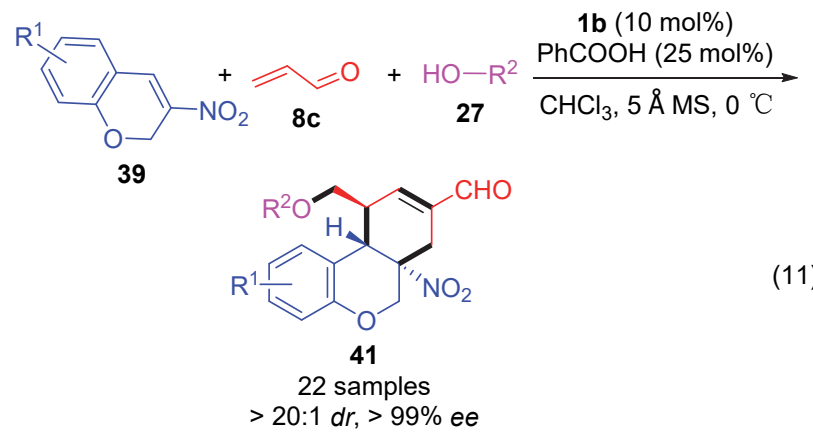




\section{3 手性哌啶衍生物的不对称合成}

\section{1 多取代手性哌啶衍生物的不对称合成}

哌啶衍生物 $42 \sim 45$ 是一类重要的催化剂和有机反 应合成子, 多取代手性哌啶是构成多种天然化学成分的 重要结构单元，广泛分布于多种天然生物碱和生物活性 成分中 ${ }^{[36]}$. 近年来, 多取代手性哌啶衍生物的合成研究 受到了普遍关注并取得了重要进展 ${ }^{[37]}$.

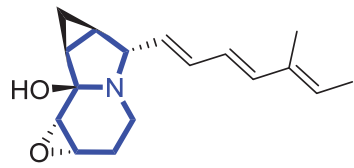

Indolizomycin (42)

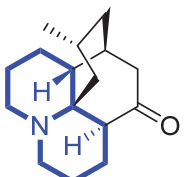

(-)-Lycopodine (44)

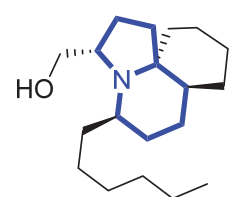

(-)-Lepadiformine (43)

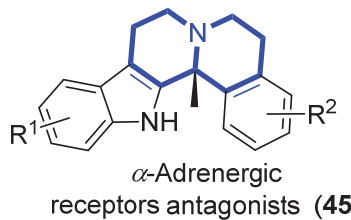

2010 年，许鹏飞研究组 ${ }^{[38]}$ 报道了二芳基脯氨醇硅 醚(1e)和双功能硫脲(13b)协同催化脂肪醛(4)、硝基烯烃 (5) 和 $N$-Tos 杂烯(46) 的三组分不对称 Michael/nitroMannich/环化串联反应，合成了一类全取代功能团化哌 啶衍生物 47 (Scheme 9). 该反应以中等的产率(47\% $71 \%$ )、突出的对映异构体选择性( $98 \%$ ～99\% ee) 合成了 一类高手性纯的目标化合物, 并可进一步衍生合成吡咯 烷酮、四氢吡啶等具有潜在应用价值的手性化合物。

同年, Urushima 等 ${ }^{[39]}$ 发展了二苯基脯氨醇甲基硅 醚(1b)催化的硝基烯 $(5)$ 、脂肪醛 $(4) 、 N$-杂烯 $(48 \mathrm{a})$ 和亲核 试剂 49 的四组分不对称 Michael/aza-Henry/缩氨/烯丙 基化串联反应，以 $66 \% \sim 88 \%$ 的产率，93\% 99\% ee

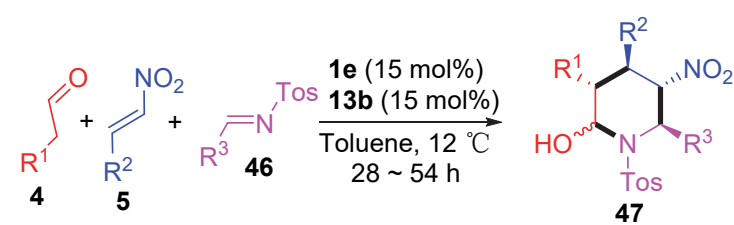

Enamine/H-bond cooperative catalysis

12 examples Cyclization reaction

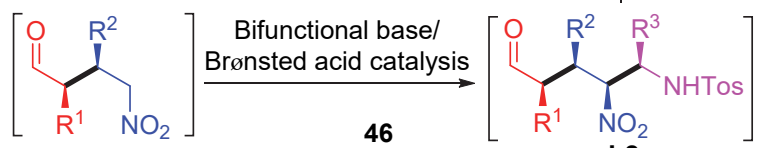

$\mathrm{I}-1$<smiles>CC(C)[C@H](CN(C)C)NC(=S)Nc1cc(C(F)(F)F)cc(C(F)(F)F)c1</smiles>

I-2

图式 9 有机催化三组分不对称 Michael/nitro-Mannich/环化串 联反应合成全取代手性哌啶化合物 47

Scheme 9 Synthesis of chiral fully substituted piperidines 47 by asymmetric organocatalytic three-component Michael/nitro-Mannich/cyclization cascade reaction

的对映异构体选择性合成了一系列多取代手性哌啶 衍生物 $\mathbf{5 0}$ (Scheme 10). 反应在 $\mathbf{1 b}$ 的仲胺催化作用下, 先后发生不对称 Michael 加成得到中间体 I 和不对称 $a z a-M i c h a e l$ 加成得到中间体 II，再经分子内缩氨化关环 反应得到中间体 III，然后与亲核试剂发生 Lewis 酸催化 的烯丙基化反应(或氰基化反应)，合成了具有 5 个手性 中心的哌啶衍生物 50. 多取代手性哌啶衍生物 50 的 硝基、氧基等可以进一步转化成其它官能团，在有机 合成及药物开发领域具有潜在的应用价值.

2012 年, Chawla 等 ${ }^{[40]}$ 进一步开发了二苯基脯氨醇 甲基硅醚(1b)催化的不对称 Michael/aza-Henry/缩氨化
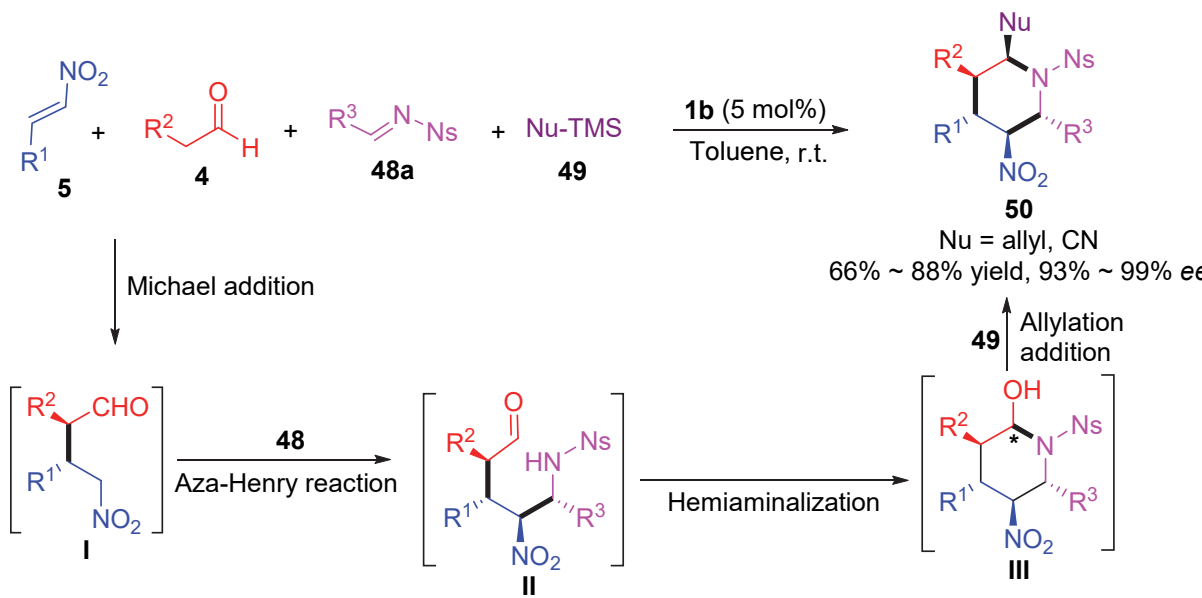

图式 10 有机催化四组分不对称 Michael/aza-Henry/缩氨/烯丙基化串联反应

Scheme 10 Organocatalytic four-component Michael/aza-Henry/hemiaminalization/allylation cascade reaction 
串联反应，报道了基于手性仲胺催化的脂肪醛(4)、硝基 烯(5)和 $N-T s$ 甲酰胺(51)的三组分不对称 $[2+2+2]$ 环化 串联反应, 实现了多取代手性 $N$-甲酰哌啶衍生物 52 的 首次合成(Eq. 12). 该研究通过手性仲胺催化的串联反 应，实现了哌啶环上五个手性中心的高效控制.

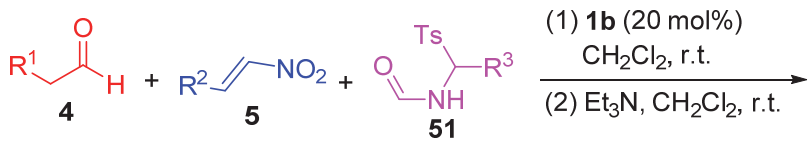

$$
\begin{aligned}
& \text { samples } \\
& 72 \% \sim 91 \% \text { yield } \\
& 84 \% \sim 97 \% \text { ee }
\end{aligned}
$$

构建手性桥环季碳中心是合成 Indolizidine 和 Guinolizidine 衍生物的关键和难点, 林国强研究组 ${ }^{[41]}$ 于 2014 年建立了以 $5 \mathrm{~mol} \%$ 的二苯基脯氨醇甲基硅醚(1b) 为催化剂、DIPEA 为碱, 建立了基于醛(4)、硝基烯烃(5) 和酮亚胺 $(\mathbf{5 3})$ 的三组分不对称串联反应, 高效地构筑了 手性桥环季碳中心, 并完成了一系列 Indolizidine 衍生 物 54 和 Guinolizidine 衍生物 55 的不对称合成(Eq. 13). 该方法可以实现克量级的产物合成, 当把反应物的量放 大到 $6 \mathrm{mmol}$ 时, 仍可获得 $85 \%$ 的产率、优异的立体选 择性( $>20: 1 d r, 96 \% e e)$.

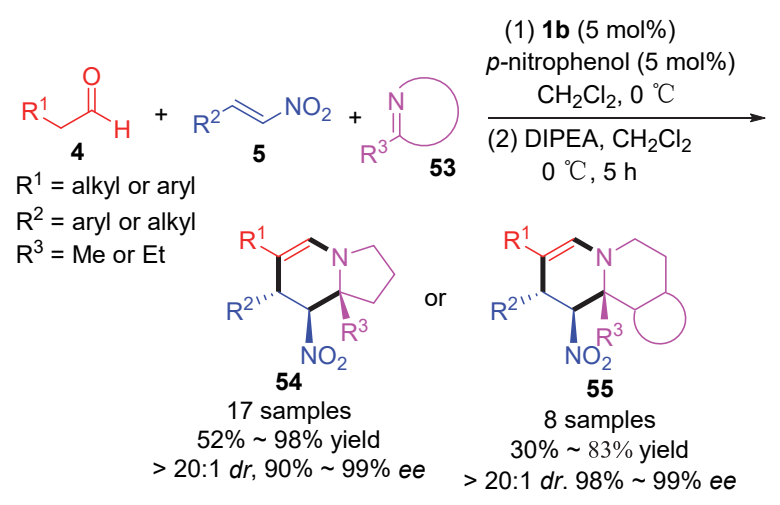

\section{2 手性喹啉衍生物的不对称合成}

多取代手性喹啉是一类重要的含氮杂环化合物, 广 泛存在于天然产物如类固醇生物碱(56)、Isoschizogaline (57) 和 Isoschizogamine (58)等分子结构中 ${ }^{[42]}$.

Rai 等 ${ }^{[43]}$ 于 2011 年建立了手性仲胺催化的三组分 “一锅法” 不对称 $[2+2+2]$ 环化串联反应合成功能团 化的十氢喹啉衍生物的新方法. 以二苯基脯氨醇甲基硅 醚(1b)为催化剂, $\mathrm{K}_{2} \mathrm{CO}_{3}$ 做碱条件下, 发生环己酮(59)和 硝基烯(5)的不对称 Michael 加成, 其加成产物与 $N$-Ts 亚
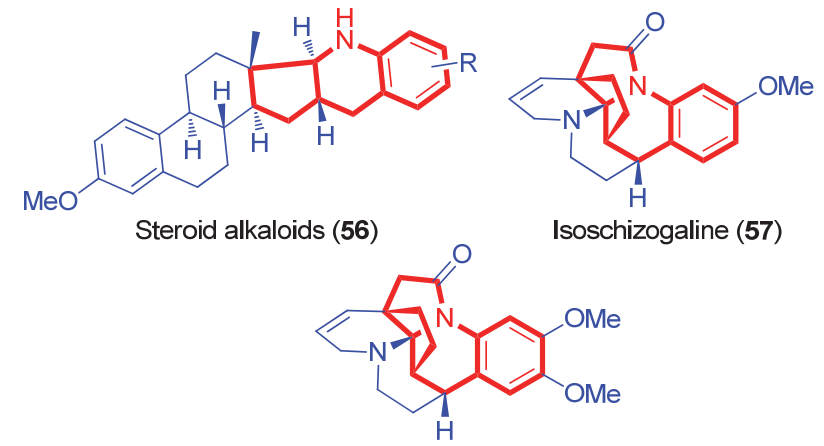

Isoschizogamine (58)

胺(48b)发生分子间的 $a z a-H e n r y$ 反应和分子内的缩氨化 反应，最终以 $69 \% \sim 94 \%$ 的产率、 $89 \% \sim 99 \%$ ee 的对映 异构体选择性合成了含有五个连续手性中心的多取代 十氢喹啉衍生物 61 (Scheme 24). 催化剂通过手性仲胺 及空间位阻效应对产物的立体选择性进行控制, 其可能 的过渡态如 TS-A 所示. 同年, Rai 等 ${ }^{[44]}$ 还探究了 1,3-环 己酮(60)在三组分不对称 Michael/aza-Henry/缩氨化串 联反应中的应用, 经 $\mathbf{1 b}$ 催化的不对称 Michael 加成经历 TS-B 所示的反应过渡态，以优异的产率(68\% 91\%)和 对映异构体选择性 $(87 \%$ ～98\% ee) 合成了一系列八氢喹 啉衍生物 62 (Scheme 11).

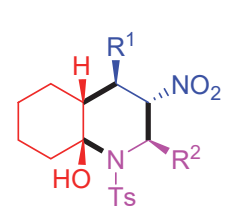

61

16 samples $69 \% \sim 94 \%$ yield $89 \% \sim 99 \%$ ee
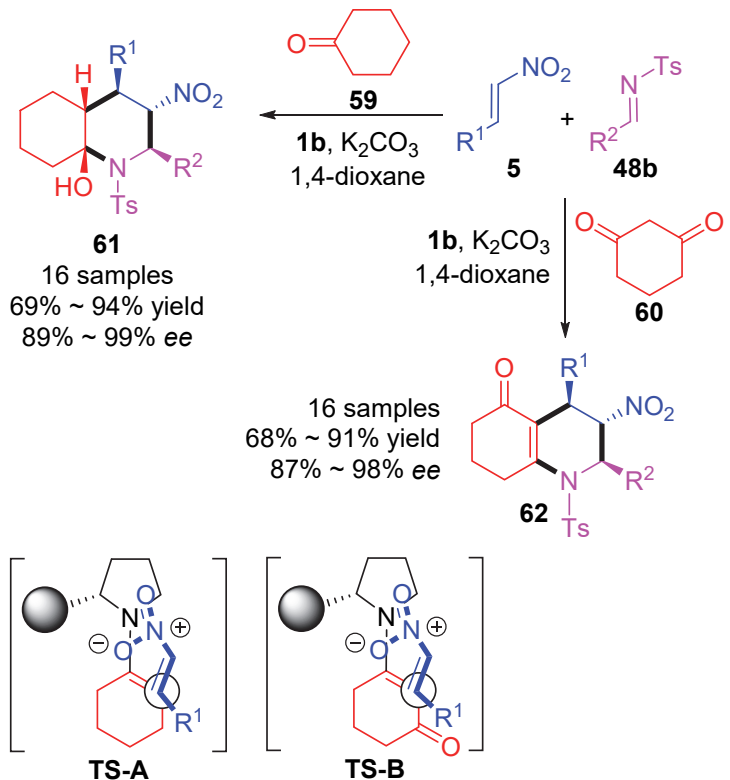

图式 11 有机催化三组分不对称串联反应合成手性喹啉衍生 物 61 和 62

Scheme 11 Synthesis of decahydroquinolines derivatives 61 and 62 by asymmetric organocatalytic cascade reaction

2011 年, Jensen 等 ${ }^{[45]}$ 建立了有机催化三组分不对称 $a z a$-Diels-Alder/rearomatization 串联反应的合成新方法. 以 $\delta, \varepsilon$-不饱和醛 $(63)$ 、硝基烯烃 $(5)$ 及苯胺衍生物 64 为起 始原料, $10 \mathrm{~mol} \%$ 的二苯基脯氨醇甲基硅醚(1b)为催化 剂, 合成了一系列具有五个手性中心的多环六氢环戊二 
烯并 $[b]$ 喹啉衍生物 65 (Eq. 14). 该研究具有产率高 $(55 \% \sim 85 \%)$ 、立体选择好( $\geqslant 19: 1 d r, 98 \% \sim 99 \% e e)$ 、 底物适用性广等特点, 为该类化合物的进一步研究应用 奠定了基础.

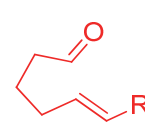

63

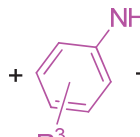

$\mathrm{R}^{3} 64$
(1) $1 \mathrm{~b}(10 \mathrm{~mol} \%)$ $\mathrm{AcOH}(20 \mathrm{~mol} \%)$ $\mathrm{CH}_{2} \mathrm{Cl}_{2}, 30^{\circ} \mathrm{C}, 16 \mathrm{~h}$

(2) $\mathrm{CH}_{2} \mathrm{Cl}_{2}, 24 \mathrm{~h}$<smiles>[R]C(C[N+](=O)[O-])[C@H]1CC[C@@H]2[C@@H]1Nc1ccccc1[C@@H]2[R7]</smiles>

65

(26 samples)

$55 \% \sim 85 \%$ yield $\geq 19: 1 d r, 98 \% \sim 99 \%$ ee

\section{4 其它类型化合物不对称合成}

\section{1 手性氢化吡喃衍生物的不对称合成}

手性四氢吡喃骨架广泛存在于具有生物活性的天 然产物和药物分子中, 开发构建手性四氢吡喃骨架的新

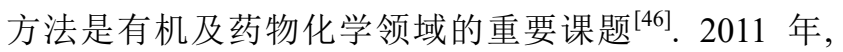
Ishikawa 等 ${ }^{[47]}$ 报道了硝基烯参与的四组分 “一锅法” 不 对称 Michael/Henry/缩醛/异构化串联反应，合成了两类 硝基手性构型相反的四氢吡喃衍生物 66,67 (Scheme 12). 该反应利用二苯基脯氨醇甲基硅醚(1b)为手性催化
剂，发生脂肪醛(4)和硝基烯(5)的不对称 Michael 加成, 得到的 Michael 加成中间体 I 与醛 $(68 \mathrm{a}$ 或 $4 d)$ 发生缩醛 反应. 该方法的主要亮点是通过碱的控制实现了化合物 的手性翻转. 当分别使用三乙胺和 1,5-二氮杂二环 [5.4.0]十一烯-5 (DBU)为碱时, 以优异的立体选择性 (91\% 99\% ee 和 94\% 99\% ee) 合成了两类硝基手性构 型相反的四氢吡喃化合物.

彭成研究组 ${ }^{[48]}$ 于 2014 年首次报道了叔胺和仲胺协 同催化的 Morita-Baylis-Hillman/Michael/缩醛化三组分 不对称串联反应. 该反应以硝基烯(5)和乙醛酸酯 $(68)$ 作 为底物, 在 $10 \mathrm{~mol} \%$ 的 4-二甲氨基吡啶(DMAP)催化作 用下形成外消旋体的加成产物 69 . 随后，通过二苯基脯 氨醇甲基硅醚(1b)催化, 69 进一步与脂肪醛(4)发生串联 反应，以良好的非对映异构体选择性(80：20～95：5 $d r)$ 和优异的对映异构体选择性(95\% 99\% ee) 合成了一 系列高度功能团化的全取代四氢吡喃衍生物 $\mathbf{7 0}$, 并以 $72 \%$ ９9\% ee 的对映异构体选择性获得了高手性纯的 醇 69 (Scheme 13). 该研究对不同的反应底物都具有良 好的普遍适用性，并成功实现了产物 69 在不对称串联 反应中的动力学拆分.

含有氢化吡喃的手性狮满酮是有机及药物合成领 域的重要分子，广泛分布于具有生物活性的天然产物和 药物分子中 ${ }^{[49]}$. 彭成研究组 ${ }^{[50]}$ 于 2013 年报道了水合菛 三酮(71)、硝基烯烃(5)与脂肪醛 (4) 的三组分不对称

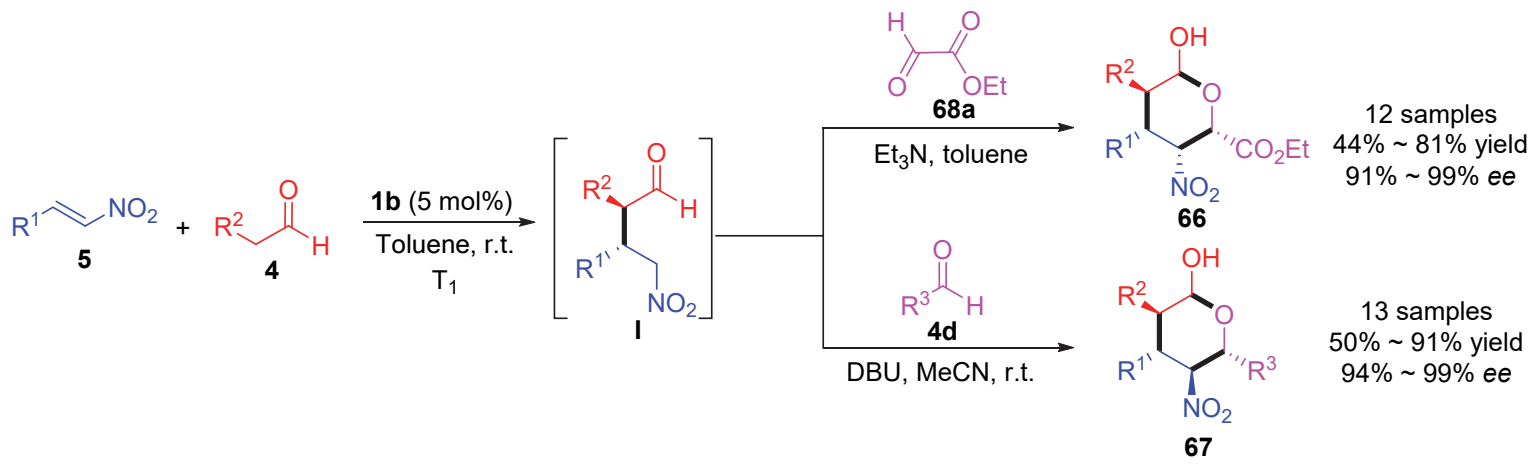

图式 12 有机催化三组分不对称 Michael/Henry/缩醛/异构化串联反应

Scheme 12 Organocatalytic three-component Michael/Henry/acetalization/isomerization cascade reaction

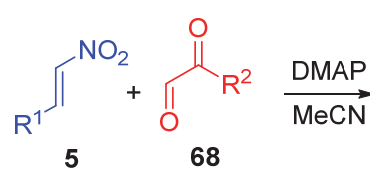

$\stackrel{\mathrm{MMCN}}{\longrightarrow}$

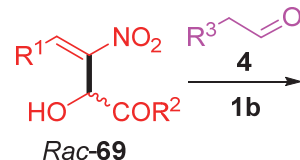

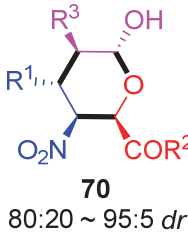
$80: 20 \sim 95: 5 d r$
$95 \% \sim 99 \%$<smiles>[R]C=C(O)C(O)O[R]</smiles>

(S)-69

$72 \% \sim 99 \%$ ee

图式 13 有机催化三组分不对称 Morita-Baylis-Hillman/Michael/缩醛化串联反应合成全取代四氢呋喃衍生物 70 Scheme 13 Synthesis of chiral fully substituted tetrahydropyrans derivatives $\mathbf{7 0}$ by asymmetric organocatalytic three-component Morita-Baylis-Hillman/Michael/acetalization cascade reaction 
Morita-Baylis-Hillman/Michael 串联反应. 以 4-氟苯 甲酸作为添加剂, 手性仲胺及三级胺协同催化, 以 $60 \% \sim 78 \%$ 的产率、83:17 92:8 $d r$ 的非对映异构体 选择性、95\% 99\% ee 的对映异构体选择性合成了一系 列手性螺环氧化狮满酮化合物 72 (Scheme 14). 研究发 现，当用 4-甲基-2-戊烯醛(4h)作为 Michael 加成受体时， 可发生双催化的不对称 MBH/Michael/缩醛/碘环化串联 反应，以良好的产率 $(61 \% \sim 71 \%$ )、优异的立体选择性 (90：10 92：8dr,94\% 98\%ee)合成具有五个连续手 性中心的手性螺环䒢满酮衍生物 73 (Scheme 14).

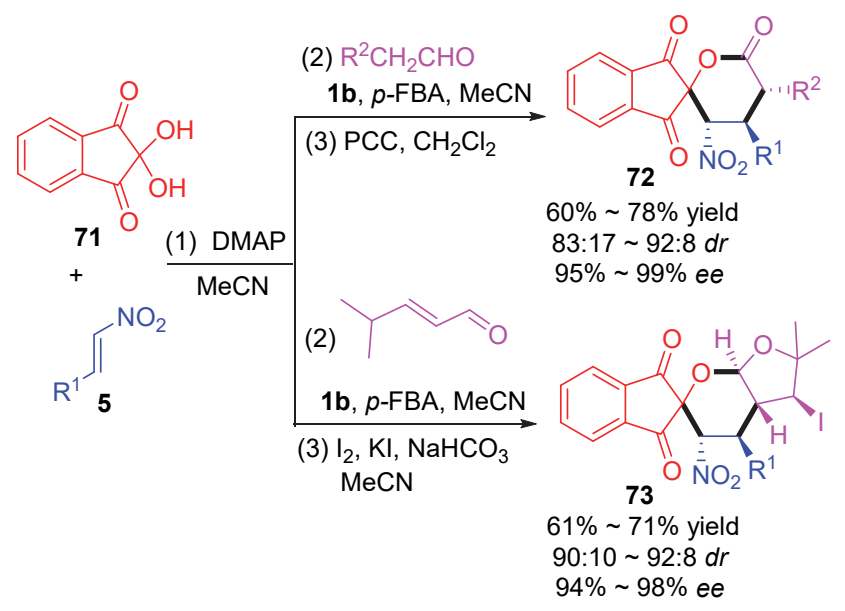

图式 14 有机催化三组分不对称串联反应合成手性螺环䒢满 酮衍生物 72 和 73

Scheme 14 Synthesis of chiral oxa-spirocyclic indanone derivatives $\mathbf{7 2}$ and $\mathbf{7 3}$ by asymmetric organocatalytic three-component cascade reaction

2016 年, Dochain 等 ${ }^{[51]}$ 报道了首例基于有机一金属协 同催化的四组分不对称 oxa-Michael/Michael/Michael/ aldol/hetero-Diels-Alder 串联反应, 合成了一类多取代三 环聚醚衍生物 74 (Eq. 15). 反应在 $5 \mathrm{~mol} \%$ 的二苯基脯胺 醇硅醚(1c)催化作用下, 发生醇 $(75)$ 、两分子丙烯醛 $(8 \mathrm{c})$ 和硝基烯(5)的四组分 oxa-Michael/Michael/Michael/aldol 串联反应，不需分离处理，再用 $20 \mathrm{~mol} \%$ 镧系配合物 $\left.[\mathrm{Yb} \text { (fod })_{3}\right]$ 为催化剂, 发生分子内逆电子杂 Diels-Alder

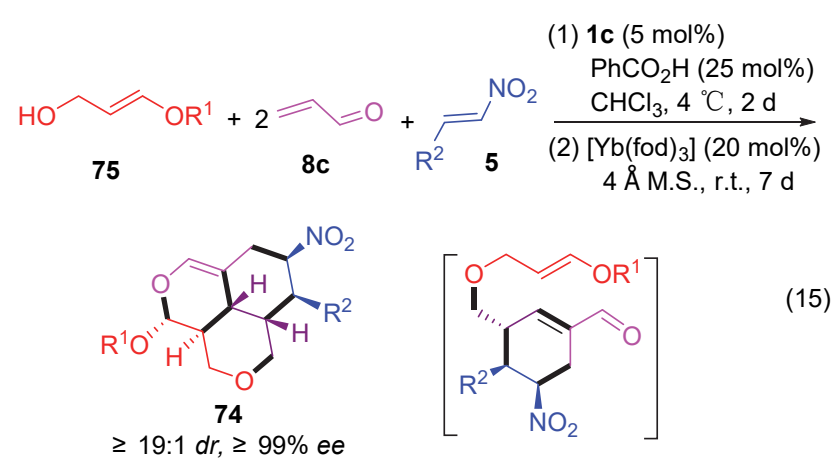

反应，以优异的立体辟了有机催化一镧系选择性 $(\geqslant 19$ : $1 d r$ 和 $\geqslant 99 \% e e)$ 实现了 6 个新的 $\mathrm{C}-\mathrm{C} / \mathrm{O}$ 键的构建和 6 个手性中心的立体选择性控制. 该研究开元素催化在有 机不对称串联合成中催化应用的新局面.

多取代手性氧杂双环[2.2.2]辛烷衍生物是一类重要 的手性氢化吡喃类杂环化合物, 广泛存在于天然产物与 生物活性药用化合物中 ${ }^{[52]}$, 具有抗菌、抗癌、消炎、镇 痛等重要的药用活性 ${ }^{[53]}$, 寻找构建手性多取代氧杂双 环[2.2.2]辛烷骨架的合成新方法受到药学、有机合成工 作者的广泛关注 ${ }^{[54]} .2016$ 年, 许丹倩研究组 ${ }^{[55]}$ 以二苯基 脯氨醇甲基硅醚(1b)为催化剂, 开发了 $\alpha, \beta$-不饱和醛 (8)、 $\alpha$-酩酰胺(75)和硝基烯烃(5)的三组分 “一锅法” 不 对称 Michael/Michael/Henry/半缩醛串联反应，以 55\% 99\%的产率、优异的立体选择性(>25：1 $d r, 86 \% \sim 99 \%$ $e e)$ 合成了 23 个多取代手性氧杂双环[2.2.2]辛烷衍生物 76 (Eq. 16). 该反应在催化剂 1 b (20 mol\%)和 4-氟苯甲 酸(10 mol\%)的作用下, 发生不对称双 Michael/Henry 串 联反应形成了中间体 IV, 再进行分子内半缩醛反应得 到具有高手性纯的目标化合物 76. 该方法通过多级不 对称串联反应，能同时对七个连续手性中心进行不对称 控制，有望开发应用于复杂天然产物的全合成.
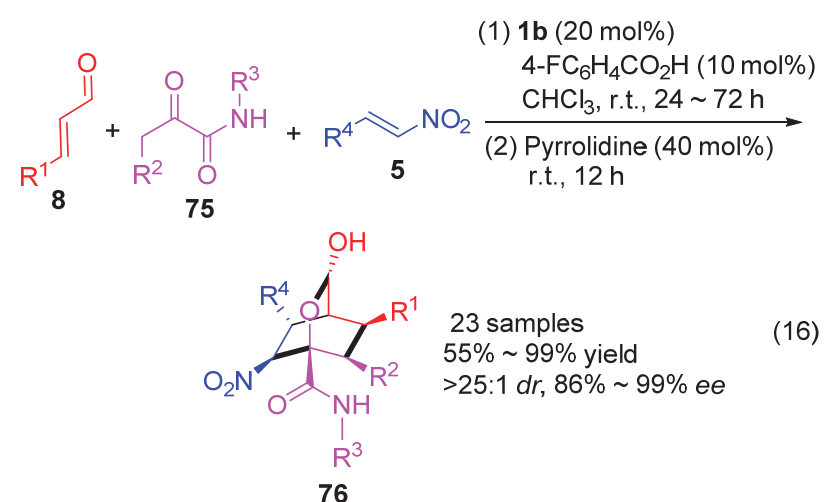

23 samples $55 \% \sim 99 \%$ yield $>25: 1 d r, 86 \% \sim 99 \%$ ee
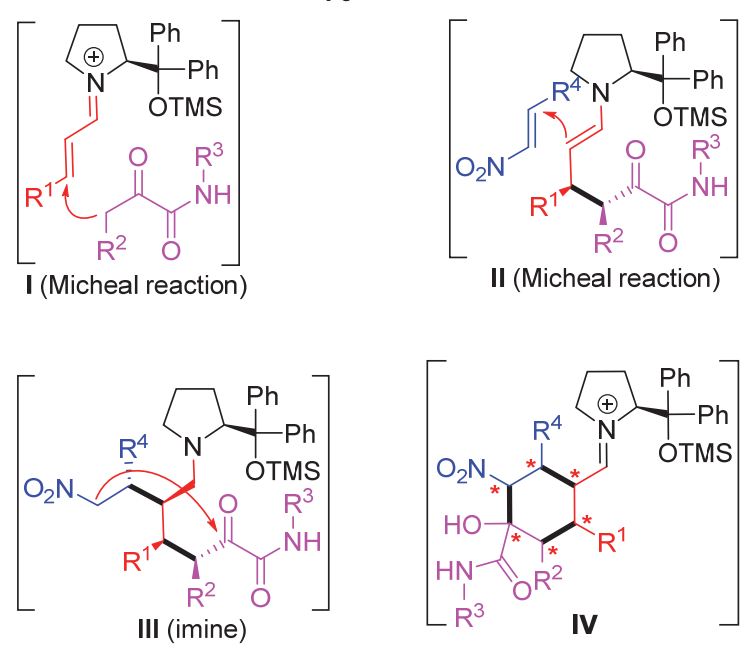


\section{2 多取代手性环烷烃衍生物的不对称合成}

手性环烷烃是构成多种药物分子及复杂天然产物 成分的基本结构单元，构建全取代手性环烷烃的多手性 中心是合成的重点和难点 ${ }^{[56]}$. 近年来基于手性仲胺催 化构建全取代手性环烷烃骨架已成为药物及有机合成 研究领域的热点课题 ${ }^{[57]} .2010$ 年, Enders 研究组 ${ }^{[58]}$ 报道 了硝基烯参与的三组分不对称 Michael/Michael/aldol/ sulfa-Michael 串联反应, 构建了含有 6 个手性中心, 具 有潜在药用价值的六氢苯并噻吩衍生物 78 (Scheme 15). 通过利用二苯基脯氨醇甲基硅醚(1b)为催化剂，发生脂 肪醛(77)和硝基烯化合物(5)的不对称 Michael 加成, 得 到的加成产物(I)与 $\alpha, \beta$-不饱和醛 $(\mathbf{8})$ 再发生 Michael 加成, 得到的中间体 II 再经分子间 aldol 缩合, 以优异的立体 选择性( $>97 ： 3 d r,>99 \% e e$ ) 得到手性环己烯醛 79, 然后在碱作用下发生 sulfa-Michael 加成得到六氢苯并噻 吩衍生物 78. 该研究为六氢苯并噻吩类化合物的进一 步药物活性研究奠定了基础.

2012 年, 王锐研究组 ${ }^{[59]}$ 开展了 Michael/Michael/ Henry 串联反应研究, 建立了二苯基脯氨醇甲基硅醚 (1b)与手性奎宁硫脲衍生物(13a)双催化的三组分串联 反应，在温和的反应条件下合成含有六个连续立体中心 的全取代手性环己烷衍生物 80 (Scheme 16). 在该合成 过程中, 脂肪醛(4)和一分子硝基烯烃(5)首先在 $1 \mathrm{~b}$ 的催 化作用发生不对称 Michael 加成反应得到中间体 I-1, 然 后经手性硫嫝-奎宁(13a)催化依次发生分子间的不对称 Michael 加成(I-2)及分子内的不对称 Henry 反应得到化 合物 80. 合成的全取代手性环己烷衍生物 80 可作为反 应中间体应用于多种复杂手性化合物的不对称合成.

2015 年, 彭成研究组 ${ }^{[60]}$ 报道了硝基烯参与的三组 分不对称 Michael/Michael/aldol 串联反应，通过 $[2+2+$ 2]环化作用成功合成了两类具有潜在生物活性的全取 代螺环己烷衍生物 81, 82 (Scheme 17). 反应在 $10 \mathrm{~mol} \%$ 二苯基脯氨醇甲基硅醚(1b)的催化作用下, 脂肪醛(4)和
硝基烯化合物(5)发生不对称 Michael 加成反应得到了手 性 $\gamma$-硝基醛中间产物，该中间体进一步与化合物 83a $83 \mathrm{c}$ 发生串联反应形成 81. 有趣的是, 当中间体与化合 物 83d 反应时, 产物的硝基 $\alpha-\mathrm{C}$ 可以实现手性翻转, 得 到与 81 构型相反的化合物 82 . 该反应具有优异的立体 选择性(90\% 99\% ee, $75: 25 \sim 95 ： 5 d r)$.

随后, Raja 等 ${ }^{[61]}$ 于 2016 年建立了环境友好型的三组 分不对称 Michael/Michael/Henry 串联反应，合成了一系 列 Hajos-Parrish-type 酮衍生物 84 (Eq. 17). 该研究以水 和乙腈 $(2: 1)$ 为溶剂, 开发了二苯基 $L$-脯氨醇(1a)催化 的 2-甲基-1,3-环戊二酮 $(85)$ 、丙烯醛 $(8 \mathrm{c})$ 和硝基烯烃(5) 的三组分不对称 “一锅法” 串联反应，以优异的对映异 构体选择性(90\% 94\% ee) 构建了五个手性中心(含两个 手性季碳中心). 在反应中, 催化剂 $1 \mathbf{a}$ 通过与羰基形成 烯胺中间体从而活化反应物，并通过位阻和氢键作用控 制产物的立体选择性. 该反应首次实现了在水相环境下 环戊二酮参与的不对称 Michael/Michael/Henry 串联反 应, 在有机化学合成中有重要的应用价值.

\section{3 手性吡唑啉酮衍生物的不对称合成}

吡唑啉酮作为一类重要的生物活性分子骨架已被 开发利用百余年，一些代表性的吡唑啉酮类药物如安替 比林、安乃近等是重要的退热止痛药 ${ }^{[62]}$, 其它的吡唑啉 酮衍生物也表现出良好的抗 HIV、抗菌等生物活性 ${ }^{[63]}$. 因此，构建吡唑啉酮骨架并合成手性吡唑啉酮衍生物已 成为药物研发、有机合成领域的热点课题. 2016 年，杜 大明研究组 ${ }^{[64]}$ 报道了二苯基 $L$-脯胺醇硅醚(1d)和双官 能团化的手性方酰胺(13c)协同催化的三组分不对称 Michael/Michael/aldol 串联反应. 该研究以吡唑啉酮类 化合物 (86)、硝基烯烃(5)和 $\alpha, \beta$-不饱和醛 (8) 为起始原料, 双催化作用下发生三级串联反应，再经氯铬酸吡啶鎓 (PCC) 连续氧化，以 $30 \% \sim 78 \%$ 的产率、突出的立体选择 性(25：1 89：11 dr, >99\%ee)合成了 16 个环己酮稠合

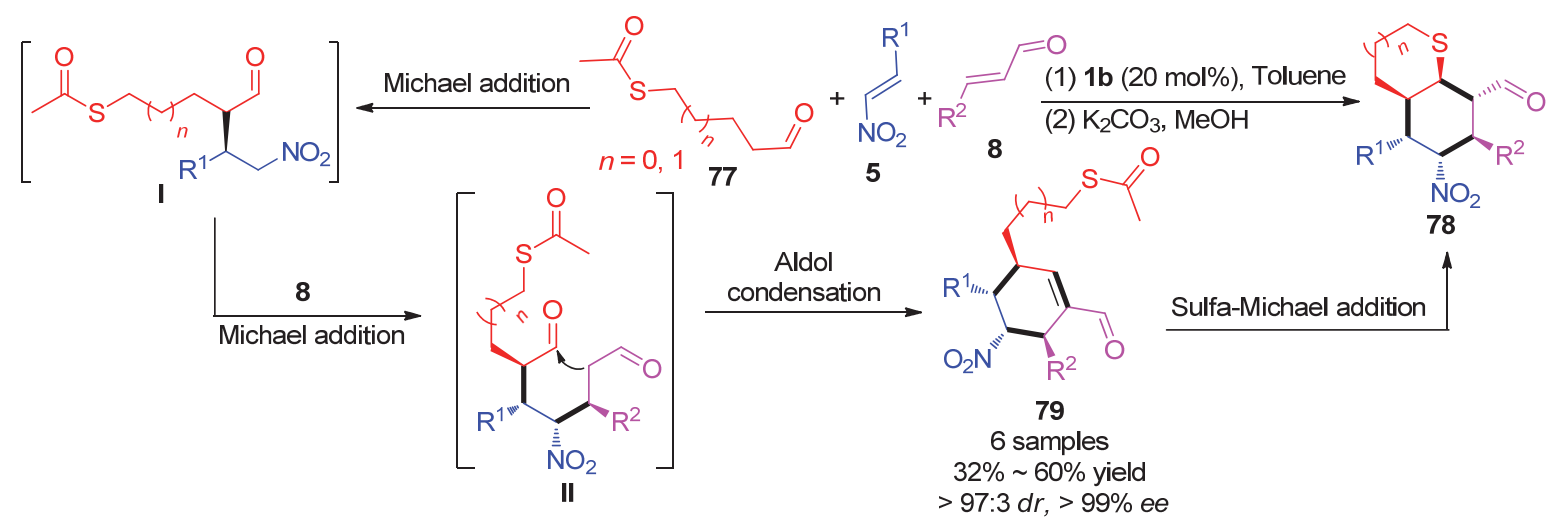

图式 15 有机催化三组分 Michael/Michael/aldol/sulfa-Michael 串联反应

Scheme 15 Organocatalytic three-component Michael/Michael/aldol/sulfa-Michael cascade reaction 


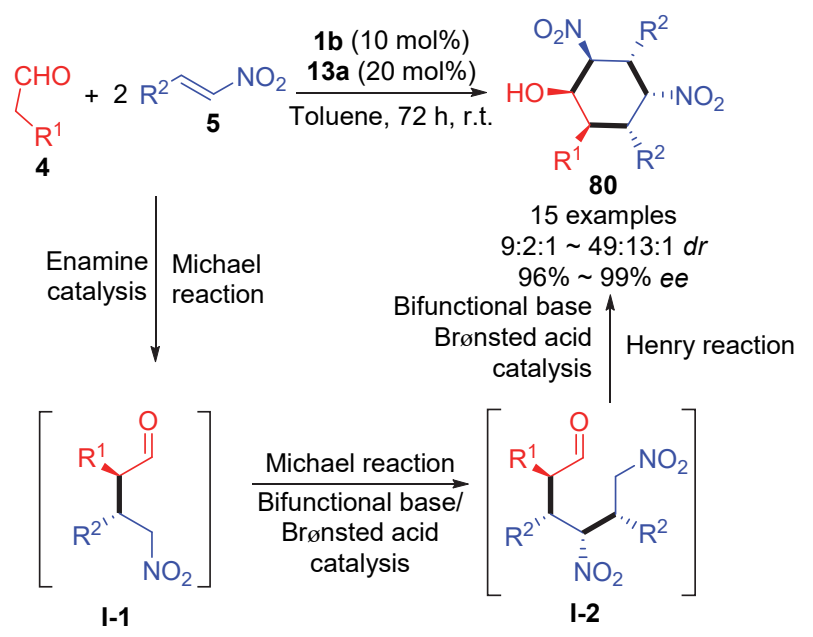

图式 16 有机催化三组分不对称 Michael/Michael/Henry 串联 反应合成全取代手性环己烷衍生物 80

Scheme 16 Synthesis of chiral fully substituted cyclohexane derivatives $\mathbf{8 0}$ by asymmetric organocatalytic three-component Michael/Michael/Henry cascade reaction

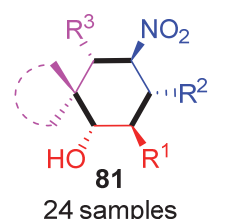

24 samples

$43 \% \sim 72 \%$ yield

$90 \% \sim 99 \%$ ee

75:25 95:5 dr

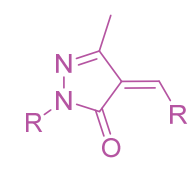

$83 a$<smiles>[R]C=C1C(=O)N(C)C(=O)N(C)C1=O</smiles>

$83 c$

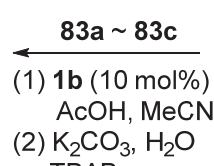
$\mathrm{TBAB}$
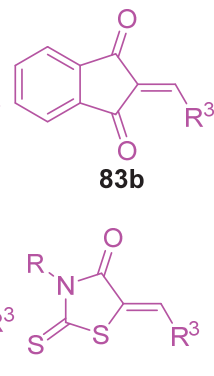

83d
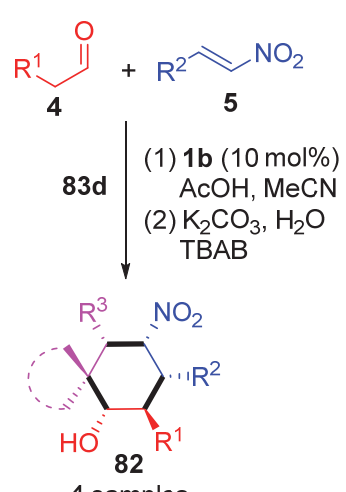

$46 \% \sim 56 \%$ yield

$90 \% \sim 99 \%$ ee

$90: 10 \sim 95: 5 d r$
图式 17 有机催化不对称 Michael/Michael/aldol 串联反应

Scheme 17 Asymmetric organocatalytic Michael/Michael/aldol cascade reaction<smiles>CC1C(=O)[CH+]CC1=O</smiles>

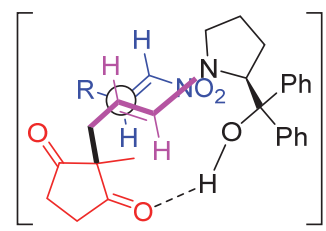

的手性螺环吡唑啉酮 87 (Eq. 18). 在串联反应过程中,化
合物 86 首先在催化剂 $13 \mathrm{c}$ 叔胺作用下经互变异构过程 发生去质子化，同时催化剂 13c 通过两个 $\mathrm{NH}$ 基团与硝 基烯(5)发生双氢键活化得到过渡态 T-A，去质子化的 13c 与硝基烯(5)发生不对称 Michael 加成. 二苯基 $L$-脯 胺醇硅醚 $(\mathbf{1 d})$ 通过亚胺离子催化 $\alpha, \beta$-不饱和酫 $(\boldsymbol{8})$ 参与的 不对称 Michael 加成, 再经分子内 aldol 反应得到目标化 合物 87. 该研究合成了一系列含有四个连续手性中心 的吡唑啉酮衍生物 87 , 为吡唑啉酮类药物的研发奠定 了基础.
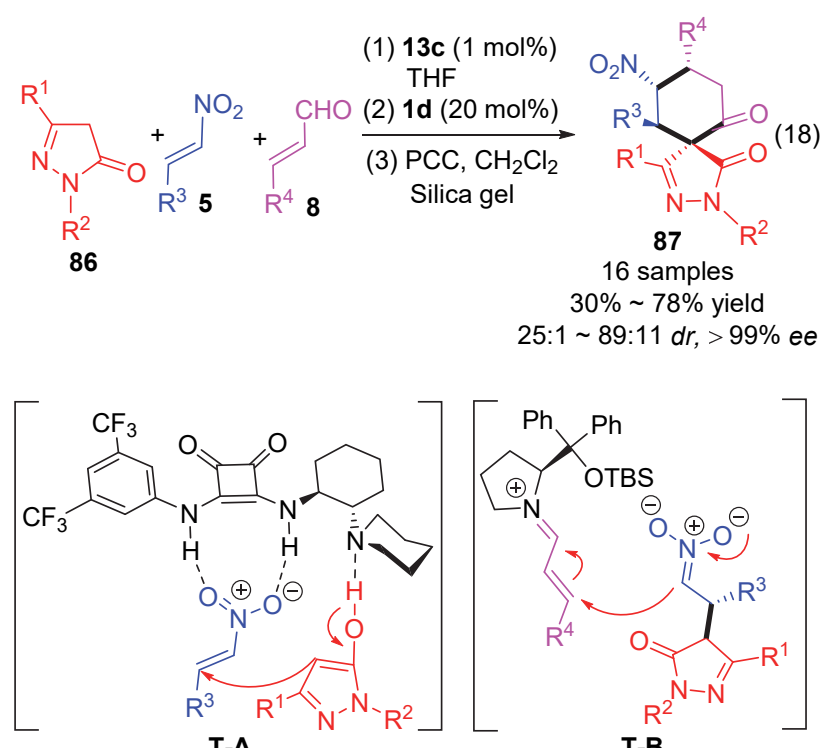<smiles>O=c1c(Nc2cc(C(F)(F)F)cc(C(F)(F)F)c2)c(N[C@@H]2CCCC[C@H]2N2CCCCC2)c1=O</smiles>

\section{5 总结与展望}

综述了以手性二芳基脯氨醇衍生物为催化剂, 硝基 烯作为反应合成子参与的多组分不对称串联合成研究. 对该研究领域下的每种合成方法分别从催化剂体系、反 应机理、实验结果、反应优点、存在的问题和局限性等 方面进行介绍. 近年来，基于手性二芳基脯氨醇衍生物 催化、硝基烯参与的多组分串联反应研究已取得较多的 成果，高效地合成了多类具有潜在生物活性和开发应用 价值的手性化合物. 但该领域仍存在很多值得深入研究 的问题和较大的挖掘空间: (1)反应类型不够丰富, 还需 发现更多新型的多组分不对称串联反应; (2)在药物开发 和天然产物不对称全合成领域的应用研究还存在很大 的空间; (3)对已合成的化合物的生物学活性研究及其在 新材料方面的应用等比较有限. 随着化学工作者对有机 反应研究的不断深入, 我们相信更多新反应、更优的反 
应体系、更多新型的手性化合物将会被研究报道，这些 新进展也将极大地推进有机催化多组分不对称串联反 应在有机合成、药物化学、材料化学等领域的研究应用.

\section{References}

[1] (a) Volla, C. M. R.; Atodiresei, I.; Rueping, M. Chem. Rev. 2014, $114,2390$.

(b) Carlone, A.; Cabrera, S.; Marigo, M.; Jørgensen, K. A. Angew. Chem., Int. Ed. 2007, 46, 1101.

(c) Enders, D.; Grondal, C.; Huettl, M. R. M. Angew. Chem., Int. Ed. 2007, 46, 1570 .

(d) Chauhan, P.; Mahajan, S.; Kaya, U.; Hack, D.; Enders, D. $A d v$. Synth. Catal. 2015, 357, 253.

(e) Yan, L. J.; Wang, Y. C. ChemistrySelect 2016, 1, 6948.

(f) Su, Y.; Bouma, M. J.; Alcaraz, L.; Stocks, M.; Furber, M.; Masson, G.; Zhu, J. Chem.-Eur. J. 2012, 18, 12624.

(g) Han, Z. Y.; Gong, L. Z. Prog. Chem. 2018, 30, 505 (in Chinese). (韩志勇, 龚流柱, 化学进展, 2018, 30, 505.)

[2] (a) Cao, Y.; Jiang, X.; Liu, L.; Shen, F.; Zhang, F.; Wang, R. Angew. Chem., Int. Ed. 2011, 50, 9124.

(b) Chi, Y.; Scroggins, S. T.; Fréchet, J. M. J. J. Am. Chem. Soc. 2008, 130, 6322.

(c) Enders, D.; Huettl, M. R. M.; Grondal, C.; Raabe, G. Nature 2006, 441,861 .

(d) Blümel, M.; Chauhan, P.; Hahn, R.; Raabe, G.; Enders, D. Org. Lett. 2014, 16, 6012.

(e) Liao, X.; Jiang, Y.; Lai, S. L.; Liu, Y. G.; Wang, S. B.; Xiong, X. Q. Chin. J. Org. Chem. 2019, 39, 668 (in Chinese).

(廖旭, 蒋岩, 赖石林, 刘源岗, 王士斌, 熊兴泉, 有机化学, 2019, 39, 668.)

[3] (a) Yu, X.; Wang, W. Org. Biomol. Chem. 2008, 6, 2037.

(b) Grondal, C.; Jeanty, M.; Enders, D. Nat. Chem. 2010, 2, 167.

(c) Jia, Z.-J.; Jiang, H.; Li, J.-L.; Gschwend, B.; Li, Q.-Z.; Yin, X.; Grouleff, J.; Chen, Y.-C.; Jørgensen, K. A. J. Am. Chem. Soc. 2011, 133, 5053.

(d) Zu, L.; Xie, H.; Li, H.; Wang, J.; Yu, X.; Wang, W. Chem.-Eur. J. 2008, 14, 6333 .

[4] (a) Eder, U.; Sauer, G.; Wiechert, R. Angew. Chem., Int. Ed. 1971, 10, 496.

(b) Hajos, Z. G.; Parrish, D. R. J. Org. Chem. 1974, 39, 1615.

[5] Marigo, M.; Wabnitz, T. C.; Fielenbach, D.; Jørgensen, K. A. Angew. Chem., Int. Ed. 2005, 44, 794.

[6] Hayashi, Y.; Gotoh, H.; Hayashi, T.; Shoji, M. Angew. Chem., Int. Ed. 2005, 44, 4212.

[7] (a) Rueping, M.; Kuenkel, A.; Tato, F.; Bats, J. W. Angew. Chem., Int. Ed. 2009, 48, 3699.

(b) Silvi, M.; Chatterjee, I.; Liu, Y.; Melchiorre, P. Angew. Chem., Int. Ed. 2013, 52, 10780.

(c) Ma, A.; Ma, D. Org. Lett. 2010, 12, 3634.

(d) Johansen, T. K.; Gómez, C. V.; Bak, J. R.; Davis, R. L.; Jørgensen, K. A. Chem.-Eur. J. 2013, 19, 16518.

(e) Talavera, G.; Reyes, E.; Vicario, J. L.; Carrillo, L. Angew. Chem., Int. Ed. 2012, 51, 4104.

(f) Li, J. L.; Zhou, S. L.; Chen, P. Q.; Dong, L.; Liu, T. Y.; Chen, Y. C. Chem. Sci. 2012, 3, 1879.

[8] (a) Barrett, A. G.; Graboski, G. G. Chem. Rev. 1986, 86, 751. (b) Yoshikoshi, A.; Miyashita, M. Acc. Chem. Res. 1985, 18, 284 (c) Zimmer, R.; Reissig, H. U. Chem. Soc. Rev. 2014, 43, 2888.

[9] (a) Ballini, R.; Marcantoni, E.; Petrini, M. J. Org. Chem. 1992, 57, 1316.

(b) Rao, P. S.; Kurumurthy, C.; Veeraswamy, B.; Kumar, G. S.; Poornachandra, Y.; Kumar, C. G.; Vasamsetti, S. B.; Kotamraju, S.; Narsaiah, B. Eur. J. Med. Chem. 2014, 80, 184.

(c) Werner, K. M.; de los Santos, J. M.; Weinreb, S. M.; Shang, M. J. Org. Chem. 1999, 64, 4865.

(d) Zhang, W. S.; Xu, W. J.; Zhang, F.; Li, Y. Chin. J. Org. Chem.
2019, 39, 1277 (in Chinese).

(张文生，许文静，张斐，李炎，有机化学， 2019, 39, 1277.)

[10] (a) Simon, M. O.; Li, C. J. Chem. Soc. Rev. 2012, 41, 1415.

(b) Hong, L.; Wang, R. Adv. Synth. Catal. 2013, 355, 1023.

(c) Wang, Y.-C.; Wang, J.-L.; Burgess, K. S.; Zhang, J.-W.; Zheng, Q.-M.; Pu, Y.-D.; Yan, L.-J.; Chen, X.-B. RSC Adv. 2018, 8, 5702.

(d) Sebahar, P. R.; Williams, R. M. J. Am. Chem. Soc. 2000, 122, 5666.

(e) Liao, F. M.; Du, Y.; Zhou, F.; Zhou, J. Acta Chim. Sinica 2018, 76,862 (in Chinese).

(廖富民，杜溢，周锋，周剑，化学学报, 2018, 76, 862.)

[11] (a) Zhao, Y.; Yu, S.; Sun, W.; Liu, L.; Lu, J.; McEachern, D.; Shargary, S.; Bernard, D.; Li. X.; Zhao, T.; Zou, P.; Sun, D.; Wang, S. J. Med. Chem. 2013, 56, 5553.

(b) Ding, K.; Lu, Y.; Nikolovska-Coleska, Z.; Qiu, S.; Ding, Y.; Gao, W.; Stuckey, J.; Krajewski, K.; Roller, P. P.;Tomita, Y.; Parrish, D. A.; Deschamps, J. R.; Wang, S. J. Am. Chem. Soc. 2005, 127, 10130 .

(c) Kumari, G.; Modi, M.; Gupta, S. K.; Singh, R. K. Eur. J. Med. Chem. 2011, 46, 1181.

(d) Arun, Y.; Saranraj, K.; Balachandran, C.; Perumal, P. T. Eur. J. Med. Chem. 2014, 74, 50.

(e) Kathirvelan, D.; Haribabu, J.; Reddy, B. S. R.; Balachandran, C.; Duraipandiyan, V. Bioorg. Med. Chem. Lett. 2015, 25, 389.

[12] (a) Cheng, D.; Ishihara, Y.; Tan, B.; Barbas III, C. F. ACS Catal. 2014, 4,743 .

(b) Tian, L.; Luo, Y. C.; Hu, X. Q.; Xu, P. F. Asian J. Org. Chem. 2016, 5,580

(c) Mei, G. J.; Shi, F. Chem. Commun. 2018, 54, 6607.

(d) Fang, X.; Wang, C.-J. Org. Biomol. Chem. 2018, 16, 2591.

[13] Jiang, K.; Jia, Z.-J.; Chen, S.; Wu, L.; Chen, Y.-C. Chem.-Eur. J. 2010, 16, 2852.

[14] Jiang, K.; Jia, Z.-J.; Yin, X.; Wu, L.; Chen, Y.-C. Org. Lett. 2010, $12,2766$.

[15] Xie, X.; Peng, C.; He, G.; Leng, H. J.; Wang, B.; Huang, W.; Han, B. Chem. Commun. 2012, 48, 10487.

[16] Zhou, B.; Yang, Y.; Shi, J.; Luo, Z.; Li, Y. J. Org. Chem. 2013, 78, 2897.

[17] Li, Z.-L.; Liu, C.; Tan, R.; Tong, Z.-P.; Liu, Y.-K. Catalysts 2016, 6 , 65.

[18] Chaudhari, P. D.; Hong, B. C.; Lee, G. H. Org. Lett. 2017, 19, 6112.

[19] (a) Rajapaksa, N. S.; McGowan, M. A.; Rienzo, M.; Jacobsen, E. N. Org. Lett. 2013, 15, 706.

(b) Granger, B. A.; Wang, Z.; Kaneda, K.; Fang, Z.; Martin, S. F. ACS Comb. Sci. 2013, 15, 379.

(c) Dounay, A. B.; Humphreys, P. G.; Overman, L. E.; Wrobleski, A. D. J. Am. Chem. Soc. 2008, 130, 5368.

(d) Nicolaou, K. C.; Edmonds, D. J.; Bulger, P. G. Angew. Chem., Int. Ed. 2006, 45, 7134.

[20] Tan, Y.; Luan, H. L.; Lin, H.; Sun, X. W.; Yang, X. D.; Dong, H. Q.; Lin, G. Q. Chem. Commun. 2014, 50, 10027.

[21] Enders, D.; Wang, C.; Mukanova, M.; Greb, A. Chem. Commun. 2010, 46, 2447.

[22] (a) Karasu, S.; Bayram, Y.; Ozkan, K.; Sagdic, O. J. Food Meas. Charact. 2019, 13, 1515

(b) Wu, X.; Yang, S.; Yu, H.; Ye, L.; Su, B.; Shao, Z. Biosci. Biotechnol. Biochem. 2019, 83, 1263.

[23] Enders, D.; Hüttl, M. R. M.; Raabe, G.; Bats, J. W. Adv. Synth. Catal. 2008, 350, 267.

[24] Enders, D.; Hüttl, M. R. M.; Runsink, J.; Raabe, G.; Wendt, B. Angew. Chem., Int. Ed. 2007, 46, 467.

[25] Zhang, F.-L.; Xu, A.-W.; Gong, Y.-F.; Wei, M.-H.; Yang, X.-L. Chem.-Eur. J. 2009, 15, 6815.

[26] Enders, D.; Krüell, R.; Bettray, W. Synthesis 2010, 567.

[27] Rueping, M.; Haack, K. L.; Ieawsuwan, W.; Sunden, H.;Blanco, M.; Schoepke, F. R. Chem. Commun. 2011, 47, 3828.

[28] Jia, Y.; Mao, Z.; Wang, R. Tetrahedron: Asymmetry 2011, 22, 2018.

[29] Erdmann, N.; Philipps, A. R.; Atodiresei, I.; Enders, D. Adv. Synth. 
Catal. 2013, 355, 847

[30] Philipps, A. R.; Fritze, L.; Erdmann, N.; Enders, D. Synthesis 2015, 47, 2377.

[31] (a) Simon-Levert, A.; Arrault, A.; Bontemps-Subielos N;Canal, C.; Banaigs, B. J. Nat. Prod. 2005, 68, 1412.

(b) Pratap, R.; Ram, V. J. Chem. Rev. 2014, 114, 10476.

(c) Tangdenpaisal, K.; Chuayboonsong, K.; Ruchirawat, S.; Ploypradith, P. J. Org. Chem. 2017, 82, 2672.

(d) Garrido, L.; Zubia, E.; Ortega, M. J.; Salva, J. J. Nat. Prod. 2002, 65, 1328.

[32] Kotame, P.; Hong, B. C.; Liao, J.-H. Tetrahedron Lett. 2009, 50, 704.

[33] Hong, B.-C.; Kotame, P.; Tsai, C.-W.; Liao, J.-H. Org. Lett. 2010 , $12,776$.

[34] Kumar, M.; Chauhan, P.; Valkonen, A.; Rissanen, K.; Enders, D. Org. Lett. 2017, 19, 3025.

[35] Kumar, M.; Chauhan, P.; Bailey, S. J.; Jafari, E.; von Essen, C.; Rissanen, K.; Enders, D. Org. Lett. 2018, 20, 1232.

[36] (a) Perreault, S.; Rovis, T. Chem. Soc. Rev. 2009, 38, 3149.

(b) Mengozzi, L.; Gualandi, A.; Cozzi, P. G. Chem. Sci. 2014, 5, 3915.

(c) Friedman, R. K.; Rovis, T. J. Am. Chem. Soc. 2009, 131, 10775.

(d) Eschenbrenner-Lux, V.; Küchler, P.; Ziegler, S.; Kumar, K.; Waldmann, H. Angew. Chem., Int. Ed. 2014, 53, 2134.

(e) Khashper, A.; Lubell, W. D. Org. Biomol. Chem. 2014, 12, 5052 .

[37] (a) Hayashi, Y.; Gotoh, H.; Masui, R.; Ishikawa, H. Angew. Chem., Int. Ed. 2008, 47, 4012.

(b) Han, B.; Li, J. L.; Ma, C.; Zhang, S. J.; Chen, Y. C. Angew. Chem., Int. Ed. 2008, 47, 9971.

(c) Han, B.; He, Z. Q.; Li, J. L.; Li, R.; Jiang, K.; Liu, T. Y.; Chen, Y. C. Angew. Chem., Int. Ed. 2009, 48, 5474

[38] Wang, Y.; Yu, D.-F.; Liu, Y.-Z.; Wei, H.; Luo, Y.-C.; Dixon, D. J.; Xu, P.-F. Chem.-Eur. J. 2010, 16, 3922.

[39] Urushima, T.; Sakamoto, D.; Ishikawa, H.; Hayashi, Y. Org. Lett. 2010, 12, 4588.

[40] Chawla, R.; Rai, A.; Singh, A. K.; Yadav, L. D. S. Tetrahedron Lett. 2012, 53, 5323 .

[41] Tan, Y.; Chen, Y. J.; Lin, H.; Luan, H. L.; Sun, X. W.; Yang, X. D.; Lin, G. Q. Chem. Commun. 2014, 50, 15913.

[42] (a) Kariba, R. M.; Houghton, P. J.; Yenesew, A. J. Nat. Prod. 2002, 65,566 .

(b) Hubbs, J. L.; Heathcock, C. H. Org. Lett. 1999, 1, 1315.

(c) Padwa, A.; Flick, A. C.; Lee, H. I. Org. Lett. 2005, 7, 2925.

(d) Zhou, J.; Magomedov, N. A. J. Org. Chem. 2007, 72, 3808.

(e) Wöfling, J.; Frank, É.; Schneider, G.; Bes, M. T.; Tietze, L. F. Synlett 1998, 1205.

(f) Mernyák, E.; Schneider, G.; Herbst-Irmer, R.; Kubas, M.; Wölfing, J. Steroids 2006, 71, 558.

[43] Rai, A.; Singh, A. K.; Singh, S.; Yadav, L. D. S. Synlett 2011, 335.

[44] Rai, A.; Singh, A. K.; Singh, P.; Yadav, L. D. S. Tetrahedron Lett. 2011, 52,1354 .

[45] Jensen, K. L.; Dickmeiss, G.; Donslund, B. S.; Poulsen, P. H.; Jørgensen, K. A. Org. Lett. 2011, 13, 3678.

[46] (a) Yeung, K.-S.; Paterson, I. Chem. Rev. 2005, 105, 4237.

(b) Nising, C. F.;Brase, S. Chem. Soc. Rev. 2012, 41, 988.

(c) Clarke, P. A., Santos S. Eur. J. Org. Chem. 2006, 2045.

(d) Larrosa, I.; Romea, P.; Urpí, F. Tetrahedron 2008, 64, 2683.

(e) Xiao, Y. Y.; Wang, Y. M.; Zhou, Z. H. Chin. J. Org. Chem. 2019, 39, 2203 (in Chinese).

(肖园园，王有名，周正洪，有机化学, 2019, 39, 2203.)

[47] Ishikawa, H.; Sawano, S.; Yasui, Y.; Shibata, Y.; Hayashi, Y. Angew. Chem., Int. Ed. 2011, 50, 3774.

[48] Han, B.; Xie, X.; Huang, W.; Li, X.; Yang, L.; Peng, C. Adv. Synth.
Catal. 2014, 356, 3676.

[49] (a) Okpekon, T.; Millot, M.; Champy, P.; Gleye, C.; Yolou, S.; Bories, C.; Loiseau, P.; Laurens, A.; Hocquemiller, R. Nat. Prod. Res. 2009, 23, 909.

(b) Seiser, T.; Cramer, N. Angew. Chem., Int. Ed. 2010, 49, 10163.

(c) Kim, S. H.; Kwon, S. H.; Park, S. H.; Lee, J. K.; Bang, H. S.; Nam, S. J.; Kwon, H. C.; Shin, J.; Oh, D. C. Org. Lett. 2013, 15, 1834.

(d) Yang, Y.; Philips, D.; Pan, S. J. Org. Chem. 2011, 76, 1902.

[50] Li, X.; Yang, L.; Peng, C.; Xie, X.; Leng, H.-J.; Wang, B.; Tang, Z.-W.; He, G.; Ouyang, L.; Huang, W.; Han, B. Chem. Commun. 2013, 49, 8692 .

[51] Dochain, S.; Vetica, F.; Puttreddy, R.; Rissanen, K.; Enders, D. Angew. Chem. 2016, 128, 16387.

[52] (a) Gao, C.; Han, L.; Zheng, D.; Jin, H.; Gai, C.; Wang, J.; Zhang, H.; Zhang, L.; Fu, H. J. Nat. Prod. 2015, 78, 630.

(b) Lee, S. H.; Tanaka, T.; Nonaka, G. I.; Nishioka, I. J. Nat. Prod. 2004, 67, 1018.

(c) Vila, R.; Mundina, M.; Muschietti, L.; Priestap, H. A.; Bandoni, A. L.; Adzet, T.; Cañigueral, S. Phytochemistry 1997, 46,1127.

[53] (a) Nagata, H.; Inagaki, Y.; Tanaka, M.; Ojima, M.; Kataoka, K.; Kuboniwa, M.; Nishida, N.; Shimizu, K.; Osawa, K. J. Periodontol. 2008, 79, 1378.

(b) Kashiwada, Y.; Nonaka, G. I.; Nishioka, I.; Chang, J. J.; Lee, K. H. J. Nat. Prod. 1992, 55, 1033.

[54] (a) De Boggiatto, M. V.; De Heluani, C. S.; De Fenik, I. J.; Catalan, C. A. J. Org. Chem. 1987, 52, 1505.

(b) Wei, G. L.; Rosazza, J. P. Tetrahedron Lett. 1990, 31, 2833.

(c) Peters, R.; Fischer, D. F. Angew. Chem., Int. Ed. 2006, 45, 5736.

[55] Xia, A.-B.; Pan, G.-J.; Wu, C.; Liu, X.-L.; Zhang, X.-L.; Li, Z.-B.; Du, X.-H.; Xu, D.-Q. Adv. Synth. Catal. 2016, 358, 3155.

[56] (a) Cabrera, S.; Aleman, J.; Bolze, P.; Bertelsen, S.; Jørgensen, K. A. Angew. Chem., Int. Ed. 2008, 47, 121.

(b) Zhou, J.; List, B. J. Am. Chem. Soc. 2007, 129, 7498.

(c) Varga, S.; Jakab, G.; Drahos, L.; Holczbauer, T.; Czugler, M.; Soós, T. Org. Lett. 2011, 13, 5416.

[57] (a) Erkkilä, A.; Majander, I.; Pihko, P. M. Chem. Rev. 2007, 107, 5416.

(b) Lathrop, S. P.; Rovis, T. J. Am. Chem. Soc. 2009, 131, 13628.

(c) Scroggins, S. T.; Chi, Y.; Fréchet, J. M. Angew. Chem., Int. Ed. 2010, 49, 2393.

(d) Wang, C.; Han, Z. Y.; Luo, H. W.; Gong, L. Z. Org. Lett. 2010, $12,2266$.

[58] Enders, D.; Schmid, B.; Erdmann, N.; Raabe, G. Synthesis 2010, 2271.

[59] Mao, Z.; Jia, Y.; Xu, Z.; Wang, R. Adv. Synth. Catal. 2012, 354, 1401.

[60] Han, B.; Huang, W.; Ren, W.; He, G.; Wang, J. H.; Peng, C. Adv. Synth. Catal. 2015, 357, 561

[61] Raja, A.; Hong, B. C.; Liao, J. H.; Lee, G. H. Org. Lett. 2016, 18, 1760.

[62] (a) S Hamama, W.; G El-Gohary, H.; Kuhnert, N.; H Zoorob, H. Curr. Org. Chem. 2012, 16, 373.

(b) Horton, D. A.; Bourne, G. T.; Smythe, M. L. Chem. Rev. 2003, 103, 893.

(c) Brune, K. Acute Pain 1997, 1, 33.

[63] (a) Yoshida, H.; Yanai, H.; Namiki, Y.; Fukatsu-Sasaki, K.; Furutani, N.; Tada, N. CNS Drug Rev. 2006, 12, 9.

(b) Hadi, V.; Koh, Y. H.; Sanchez, T. W.; Barrios, D.; Neamati, N.; Jung, K. W. Bioorg. Med. Chem. Lett. 2010, 20, 6854.

(c) Chande, M. S.; Barve, P. A.; Suryanarayan, V. J. Heterocycl. Chem. 2007, 44, 49.

[64] Li, J. H.; Cui, Z. H.; Du, D. M. Org. Chem. Front. 2016, 3, 1087. 